\title{
Global Solutions to Maxwell Equations in a Ferromagnetic Medium*
}

\author{
J.L. Joly, G. Métivier and J. Rauch
}

\begin{abstract}
We study the Cauchy problem for the Landau-Lifschitz model in ferromagnetism without exchange energy. Once existence of global finite energy solutions is obtained, we study additional uniqueness and regularity properties of these solutions.
\end{abstract}

\section{Introduction}

Recently the Cauchy problem for some nonlinear models in electromagnetism have been shown to possess large finite energy solutions, by which is meant solutions satisfying the fundamental physical energy estimates, such as the electromagnetic energy and some additional conservative or dissipative estimates on new components of the electromagnetic field such as the polarization $P$ or the magnetization $M$ of the medium.

The physical estimates hinted at are 0-order with respect to derivatives. Hence, from a mathematical point of view, the above mentioned problems should be called weak Cauchy problems if one recalls the general Cauchy nonlinear problem is well-posed under stronger regularity assumptions on data, including $L^{2}$ control on derivatives up to an order that depends on the space dimension and is always greater than 0 . Instead, due to special structure of the nonlinearities, the problems we mention obey strong continuity properties that mix the properties of the nonlinearities and some geometric properties of the differential operator. It allows a mathematical analysis of the solutions leading to existence, uniqueness and regularity properties, which recalls what identifies the so-called strong Cauchy problem. This is the reason why "weak solution" is not to be found in the title of these papers.

The papers mentioned above deal with nonlinear optics in nonmagnetic mediums where the relation $D=E+P$ involves the polarization $P$ of the electric medium modeling the interaction between medium and light which, due to large intensity of the electric field $E$, is nonlinear. The anharmonic oscillator model is examined in [JMR 1], [DR] being addressed to the Maxwell-Bloch quantic model

\footnotetext{
* Research partially supported by the U.S. National Science Foundation, U.S. Office of Naval Research, and the NSF-CNRS cooperation program under grants number NSF-DMS-9203413 and OD-G-N0014-92-J-1245 NSF-INT-9314095 respectively, and the CNRS through the Groupe de Recherche G1180 POAN.
} 
which introduces a larger set of components, involving the density of exited electrons.

In contrast the present work concerns magnetic mediums where, in a suitable system of units, $D=E$ and the magnetic induction $B=H+M$ involves the magnetization $M$ which satisfies a differential equation with a source term which is nonlinear in $M$ and $H$. The so-called Landau-Lifschitz model (see [LL 1] [LL 2]) for the propagation of the electromagnetic field in a ferromagnetic medium uses the nonlinear interaction term

$$
F(m, h):=\frac{\gamma}{1+\alpha^{2}}\left(m \wedge h+\frac{\alpha}{|m|}(m \wedge(m \wedge h))\right), \quad h, m \in \mathbb{R}^{3},
$$

where $\gamma$, the gyromagnetic constant, and $\alpha$, the damping factor, are positive constants. In (1.1) the variables $m$ and $h$ need to be replaced by, respectively, the polarization $M$ and the magnetic field $H$.

The aim of this paper is to prove the existence of global finite energy solutions to the corresponding Maxwell system

$$
\left\{\begin{aligned}
\partial_{t} E-\operatorname{curl} H & =0 \\
\partial_{t} H+\operatorname{curl} E & =-\partial_{t} M \\
\partial_{t} M & =F(M, H)
\end{aligned}\right.
$$

with the usual divergence free conditions for $E$ and $B$

$$
\operatorname{div} E=\operatorname{div}(H+M)=0 .
$$

Note that the divergence free condition (1.3) is satisfied as soon as it is satisfied at $t=0$, since (1.2) immediately implies that

$$
\partial_{t}(\operatorname{div} E(t))=\partial_{t}(\operatorname{div}(H(t)+M(t)))=0 .
$$

Functions $E, H$ and $M$ denote $\mathbb{R}^{3}$-valued functions of the time-space variables $(t, x) \in \mathbb{R}^{1+3}$. The electromagnetic field is $(E, H)$ and $M$ is the magnetization of the ferromagnetic medium. $F$ is given by (1.1) or can be a more general interaction, see $\S 2$ below.

We also study the uniqueness and regularity properties of the energy solutions. With suitable assumptions on the nonlinearity $F$, we show that if the Cauchy data are smooth, then the solution remains smooth for all time. Uniqueness is proved for solutions with $(\operatorname{curl} E, \operatorname{curl} H)$ in $L^{2}$. The uniqueness of energy solutions remains an open problem. The precise results are presented in the next section.

Before stating the main results let us say more about the model (see [JMR3] for more complete discussion and bibliography). The nonlinearity we have chosen in (1.1) is far from complete. A more complete version consists in replacing $F(M, H)$ in the third equation of $(1.2)$ by $F\left(M, H+H_{\text {eff }}\right)$ with

$$
H_{e f f}=H_{s}+H_{a}+H_{e},
$$


where $H_{s}=H_{s}(x)$ is given independent of $t, H_{a}=-k(p \cdot M) p, k \geq 0, p$ a given unit vector in $\mathbb{R}^{3}$ and $H_{e}=-k^{\prime} 1_{\Omega} \triangle M, k^{\prime} \geq 0$ where $\Omega$ is an open subset of $\mathbb{R}^{3}$ such that $\bar{\Omega}=\operatorname{support} M$. The case $H_{e} \neq 0$ is completely different [V], [CF]. In particular, the equations are no longer hyperbolic. In this paper we consider only the hyperbolic case, and for simplicity we assume that $H_{\text {eff }}=0$. The terms $H_{s}$ and $H_{a}$ can be treated with minor modifications. Also note that the Cauchy problem for (1.2) is solved in space dimension one, in [JV1], [JV2].

\section{Statement of the main results}

First, we detail the properties required for the function $F$ which appears in (1.2). Assumption 2.1 $F$ is a $C^{\infty}$ function on $\mathbb{R}^{3} \times \mathbb{R}^{3}$ with values in $\mathbb{R}^{3}$, such that

$$
\begin{aligned}
& h \mapsto F(m, h) \text { is linear, } \quad m \in \mathbb{R}^{3} \\
& F(m, h) \cdot m=0, \quad m, h \in \mathbb{R}^{3} \\
& F(m, h) \cdot h \leq 0, \quad m \in \mathbb{R}^{3}
\end{aligned}
$$

If $F$ satisfies Assumption 2.1 there exists a function $C(R)$ such that for all $R>0$,

$$
\left\{\begin{aligned}
\left|F\left(m^{\prime}, h\right)-F(m, h)\right| & \leq C(R)\left|m^{\prime}-m\right||h|, & & |m| \leq R,\left|m^{\prime}\right| \leq R \\
|F(m, h)| & \leq C(R)|h|, & & |m| \leq R .
\end{aligned}\right.
$$

Assumption 2.2 $F \in C^{\infty}\left(\mathbb{R}^{3} \backslash\{0\} \times \mathbb{R}^{3} ; \mathbb{R}^{3}\right)$ satisfies (2.1) (2.2) (2.3) and the inequalities (2.4) hold for $m, m^{\prime} \neq 0$.

The function given by (1.1) with $\alpha=0$ or the function

$$
F(m, h):=\frac{\gamma}{1+\alpha^{2}}\left(m \wedge h+\frac{\alpha}{\sqrt{\delta^{2}+|m|^{2}}}(m \wedge(m \wedge h))\right), \quad h, m \in \mathbb{R}^{3}, \delta>0
$$

satisfy Assumption 2.1 whereas the function (1.1) with $\alpha>0$, which is homogeneous of degree one in $m$, is not $C^{1}$ at $m=0$ but satisfies Assumption 2.2.

Let us now state what we mean by finite energy solution.

Definition 2.3 We say that $U=(E, H, M)$ is a finite energy solution in $\Omega_{T}=$ $[0, T] \times \mathbb{R}^{3}$ if all components $E, H, M$ belong to $C^{0}\left([0, T] ; L^{2}\left(\mathbb{R}^{3}\right)\right), M$ is in $L^{\infty}\left([0, T] \times \mathbb{R}^{3}\right)$ and if $U$ is a distributional solution to (1.2) (1.3).

Proposition 2.4 Any finite energy solution satisfies

$$
\int_{\mathbb{R}^{3}}\left(|E(t, x)|^{2}+|H(t, x)|^{2}\right) d x \leq \int_{\mathbb{R}^{3}}\left(|E(0, x)|^{2}+|H(0, x)|^{2}\right) d x
$$


and

$$
|M(t, x)|=|M(0, x)| \text { a.e. }
$$

Proof. Replacing $\partial_{t} M$ by $F(M, H)$ in the second equation in (1.2) shows that a finite energy solution $(E, H)$ is the solution of a linear first order symmetric hyperbolic system with source term in $L^{1}\left([0, T] ; L^{2}\left(\mathbb{R}^{3}\right)\right)$ hence it satisfies the usual energy identities. With (2.3) this implies that

$$
\int_{\mathbb{R}^{3}}\left(|E(t, x)|^{2}+|H(t, x)|^{2}\right) d x, \quad 0 \leq t \leq T
$$

decreases in time. Similarly, $\partial_{t} M=F(M, H) \in L^{1}\left([0, T] ; L^{2}\left(\mathbb{R}^{3}\right)\right)$ and $\partial_{t} M \cdot M=0$ by $(2.2)$. Therefore

$$
|M(t, x)|, \quad 0 \leq t \leq T
$$

is time-invariant. Summing up, the quantity

$$
n_{0}(t)=\sqrt{\|E(t)\|_{2}^{2}+\|H(t)\|_{2}^{2}}+\|M(t)\|_{L^{2} \cap L^{\infty}} .
$$

satisfies

$$
n_{0}(t) \leq n_{0}(0), \quad 0 \leq t \leq T
$$

Remark. Condition (2.3) is not necessary for the validity of the theorems to be stated below. It only simplifies some of the estimates. It insures dissipativity as observed in (2.5). If (2.3) is suppressed in Assumptions 2.1 and 2.2, one has in place of (2.5)

$$
\int_{\mathbb{R}^{3}}\left(|E(t, x)|^{2}+|H(t, x)|^{2}\right) d x \leq e^{C t} \int_{\mathbb{R}^{3}}\left(|E(0, x)|^{2}+|H(0, x)|^{2}\right) d x
$$

with some constant $C$ depending only on $\left\|M_{0}\right\|_{\infty}$.

Notation. $\mathbf{L}_{\mathbf{0}}$ denotes the space of Cauchy data $U_{0}=\left(E_{0}, H_{0}, M_{0}\right) \in L^{2}\left(\mathbf{R}^{3}\right)$ such that $\operatorname{div} E_{0}=\operatorname{div}\left(H_{0}+M_{0}\right)=0$ and $M_{0} \in L^{\infty}\left(\mathbf{R}^{3}\right)$.

Theorem 2.5 Suppose that $F$ satisfies Assumption 2.2. Then the Cauchy problem for (1.2) with initial data $U_{0} \in \mathbf{L}_{\mathbf{0}}$ has a finite energy solution on $\Omega_{\infty}=$ $\left[0,+\infty\left[\times \mathbf{R}^{3}\right.\right.$.

Moreover, suppose that $\mathbf{U}_{\mathbf{0}}$ is a bounded subset of $\mathbf{L}_{\mathbf{0}}$ which is compact in $\left(L^{2}\left(\mathbf{R}^{3}\right)\right)$. Then the set $\mathbf{U}$ of finite energy solutions on $\Omega_{\infty}$, with Cauchy data in $\mathbf{U}_{\mathbf{0}}$ is compact in $\left(C^{0}\left([0, T] ; L^{2}\left(\mathbf{R}^{3}\right)\right)\right)^{3}$ for all $T>0$. 
The solutions are constructed as limits of solutions of reguralized equations. For all $\lambda>1$ define

$$
S^{\lambda}=\varphi\left(\lambda^{-1} D_{x}\right)
$$

where $\varphi \in C_{0}^{\infty}$ is a cut-off function supported by $|\xi| \leq 2$, equal to 1 on $|\xi|=1$ and such that $0 \leq \varphi \leq 1$. Consider the following approximation of the Cauchy problem for $(1.2)$.

$$
\left\{\begin{aligned}
\partial_{t} E^{\lambda}-\operatorname{curl} H^{\lambda} & =0 \\
\partial_{t} H^{\lambda}+\operatorname{curl} E^{\lambda} & =-S^{\lambda} F\left(M^{\lambda}, H^{\lambda}\right) \\
\partial_{t} M^{\lambda} & =F\left(M^{\lambda}, H^{\lambda}\right)
\end{aligned}\right.
$$

with initial conditions

$$
E_{0}^{\lambda}=S^{\lambda} E_{0}, H_{0}^{\lambda}=S^{\lambda} H_{0}, M_{0}^{\lambda}=M_{0} .
$$

Theorem 2.6 Let $F$ satisfy Assumption 2.2 and $U_{0} \in \mathbf{L}_{\mathbf{0}}$. Then, for each $\lambda \geq 1$, the Cauchy problem (2.10), (2.11) has a unique global solution $U^{\lambda}$ which belongs to $C^{1}\left(\left[0,+\infty\left[; H^{s} \times H^{s} \times L^{\infty}\right)\right.\right.$ for all s. Moreover $U^{\lambda}$ has a subsequence which converges in $C^{0}\left([0, T] ; L^{2} \times L^{2} \times L^{\infty}\right)$ for all $T>0$ to a global finite energy solution $U^{\infty}$ of (1.2) with initial data $U_{0}$.

The regularized system (2.10) has been chosen so that the two conservations laws (2.5) and (2.6) hold. This explains why there is no $S^{\lambda}$ in front of $F$ in the right hand side of the third equation. Other regularizations having this property could be considered. Thus the family of solutions $U^{\lambda}$ is bounded in $C^{0}\left(\left[0, \infty\left[; L^{2}\left(\mathbf{R}^{3}\right)\right)\right.\right.$ and $M^{\lambda}$ is bounded in $L^{\infty}$. Therefore there are subsequences which converge weakly. The difficulty is to pass to the limit in the nonlinear term $F\left(M^{\lambda}, H^{\lambda}\right)$. The main point in the proof is to show that if a subsequence $U^{\lambda}$ converges weakly, then it converges strongly, and therefore the limit is a finite energy solution. This argument also accounts for the compactness result stated in Theorem 2.5. Note that Theorems 2.5 and 2.6 do not depend specifically on the space dimension 3 . Analogous results could be proved in higher dimension. The proofs are given in sections 3 and 4.

Next we study the smoothness and uniqueness of the finite energy solutions. The components of $U$ do not behave all the same : some are propagated at speed 1 , some are propagated at speed 0 . Introduce the orthogonal decomposition of $L^{2}\left(\mathbf{R}^{3}\right)$

$$
L^{2}\left(\mathbf{R}^{3}\right)=L_{\|}^{2}\left(\mathbf{R}^{3}\right) \oplus L_{\perp}^{2}\left(\mathbf{R}^{3}\right) .
$$

Functions in $L_{\|}^{2}$ satisfy curl $=0$ whereas those in $L_{\perp}^{2}$ are such that div $=0$. The corresponding projectors are denoted by $P_{\|}: L^{2} \rightarrow L_{\|}^{2}$ and $P_{\perp}: L^{2} \rightarrow L_{\perp}^{2}$, the same notation being used in $L^{p}, 1<p<+\infty$. They are Fourier multipliers 
with symbols $\frac{1}{|\xi|^{2}}(\xi, \cdot) \xi$ and $-\frac{1}{|\xi|^{2}} \xi \wedge(\xi \wedge \cdot)$ respectively. If $U=(E, H, M)$ is a finite energy solution on $\Omega_{T}$ (Definition 2.3), it follows from $\operatorname{div} E(t)=0$ and $\operatorname{div}(H(t)+M(t))=0$ that $E_{\|}(t)=0$ and $(H(t)+M(t))_{\|}=0$. Thus, $E$ and $H$ have the orthogonal decomposition

$$
E(t)=E_{\perp}(t), \quad H(t)=H_{\perp}(t)-M_{\|}(t) .
$$

The fields $M$ and $H_{\|}$are propagated at speed 0 , while $E_{\perp}=E$ and $H_{\perp}$ satisfy a wave equation. More precisely one can extract from (1.2) a linear system for $\left(E_{\perp}, H_{\perp}\right)$ with source term and coefficients of the zero-th order term depending on $M$ and $M_{\|}$. This point of view is developed in section 5. A remarkable fact is that, for $\mu \leq 1, H^{\mu}$ regularity for $\left(E_{\perp}, H_{\perp}\right)$ is propagated from the initial data, without assuming the same regularity for $M$. This is partly due to the fact that $\partial_{t} M \in L^{2}$, while $\partial_{t}$ is not characteristic for the system satisfied by $\left(E_{\perp}, H_{\perp}\right)$. The actual proof relies on the use of Strichartz inequalities, for which the space dimension 3 is critical.

Theorem 2.7 Let $F$ satisfy Assumption 2.2. Let $U_{0} \in \mathbf{L}_{\mathbf{0}}$ be such that $E_{0}$ and $H_{0 \perp}$ belong to $H^{\mu}\left(\mathbf{R}^{3}\right)$, where $\left.\left.\mu \in\right] 0,1\right]$. Consider on $\Omega_{\infty}$ a finite energy solution $U$ with initial data $U_{0}$. Then $E$ and $H_{\perp}$ belong to $C^{0}\left(\left[0,+\infty\left[; H^{\mu}\left(\mathbf{R}^{3}\right)\right)\right.\right.$.

The next theorem completes Theorem 2.7 when $\mu=1$. It is proved in section 6 .

Theorem 2.8 Let $F$ satisfy Assumption 2.2. Let $U_{0} \in \mathbf{L}_{\mathbf{0}}$ be such that curl $E_{0}$ and curl $H_{0}$ belong to $L^{2}\left(\mathbf{R}^{3}\right)$. Then there exists a unique finite energy solution on $\Omega_{\infty}$ satisfying the Cauchy condition $U_{\mid t=0}=U_{0}$. Furthermore curl $E$ et curl $H$ belong to $C^{0}\left(\left[0,+\infty\left[; L^{2}\left(\mathbf{R}^{3}\right)\right)\right.\right.$.

The propagation of the $L^{2}$ regularity of curl $E$ and $\operatorname{curl} H$ follows from Theorem 2.7. The uniqueness is a consequence of a stronger result about the $L^{2}$ stability of such solutions. When $H \in L^{\infty}$, uniqueness and $L^{2}$ stability are trivial. For general finite energy solution, it seems difficult to get such an $L^{\infty}$ bound. First, the projector $P_{\|}$does not act in $L^{\infty}$, and all we can insure is that $H_{\|} \in L^{p}$ for all finite $p$. Second, $H_{\perp}$ satisfies a wave equation, for which $L^{\infty}$ bounds are not an easy matter. However, when $\left(E, H_{\perp}\right) \in H^{1}$, one has $\square H_{\perp} \in L^{1}\left([0, T] ; L^{2}\right)$, and estimating the $L^{2}\left(L^{\infty}\right)$ norm of $H_{\perp}$ by the $L^{1}\left(L^{2}\right)$ norm of $\square H_{\perp}$ is just the forbidden limit case of Strichartz estimates in space dimension 3. Proposition 6.3 is a substitute for these estimates. It is proved in section 8. Finally, the conclusion is that $H$ is "almost" $L^{\infty}$ and this is the key for Theorem 2.8 .

In section 7 , we study the higher order regularity of solutions.

Theorem 2.9 Let $F$ satisfy Assumption 2.1 and let $s \geq 2$. If $U_{0} \in \mathbf{L}_{\mathbf{0}} \cap H^{s}\left(\mathbf{R}^{3}\right)$ then the unique finite energy solution with initial data $U_{0}$ belongs to $C^{0}\left(\left[0,+\infty\left[; H^{s}\left(\mathbf{R}^{3}\right)\right)\right.\right.$. 
For the nonlinear function $F$ to act in $H^{2}$, it must be smooth enough. This explains why Assumption 2.1 is required there.

\section{Existence of energy solutions}

This section is devoted to the proof of Theorems 2.5 and 2.6. First we prove the existence and uniqueness of solutions to the approximate equations (2.10).

Proposition 3.1 Let F satisfy Assumption 2.2 and Cauchy data $U_{0}$ belong to $\mathbf{L}_{\mathbf{0}}$. Then, for all $\lambda \geq 1$, the Cauchy problem (2.10) (2.11) has a unique global solution $U^{\lambda}$ which belongs to $C^{1}\left(\left[0,+\infty\left[; H^{s} \times H^{s} \times L^{\infty}\right)\right.\right.$ for all $s$. Moreover there exist $C>0$ independent of $\lambda$ such that, for all $\lambda \geq 1$, the following estimates hold

$$
\begin{aligned}
\left|M^{\lambda}(t, x)\right| & =\left|M_{0}(x)\right|, \quad \text { a.e. } \\
\left\|E^{\lambda}(t)\right\|_{L^{2}}^{2}+\left\|H^{\lambda}(t)\right\|_{L^{2}}^{2} & \leq e^{C t}\left(\left\|E_{0}^{\lambda}\right\|_{L^{2}}^{2}+\left\|H_{0}^{\lambda}\right\|_{L^{2}}^{2}\right) \\
\operatorname{div} E^{\lambda}(t) & =0 \\
\operatorname{div}\left(H^{\lambda}(t)+S^{\lambda} M^{\lambda}(t)\right) & =0
\end{aligned}
$$

Proof. For $U:=(E, H, M)$ define

$$
G^{\lambda}(U):=\left(\operatorname{curl} H,-\operatorname{curl} E-S^{\lambda} F(M, H), F(M, H)\right),
$$

so that the Cauchy problem $(2.10),(2.11)$ reads

$$
\frac{d}{d t} U^{\lambda}(t)=G^{\lambda}\left(U^{\lambda}(t)\right), \quad U(0)=\left(E_{0}^{\lambda}, H_{0}^{\lambda}, M_{0}\right)
$$

Let $L_{\lambda}^{2}$ denote the closed linear subspace of $L^{2}$ of functions $u$ satisfying supp $\hat{u} \subset$ $\{|\xi| \leq 2 \lambda\}$. One has $L_{\lambda}^{2} \subset H^{s}$ for all $s$. The space $L_{\lambda}^{2}$ is equipped with the scalar product of $L^{2}$ and satisfies $L_{\lambda}^{2} \subset L^{\infty}$ with a continuous injection

$$
\|u\|_{L^{\infty}} \leq(2 \lambda)^{3 / 2}\|u\|_{L^{2}}, u \in L_{\lambda}^{2} .
$$

1) We first show that $G^{\lambda}$ maps $L_{\lambda}^{2} \times L_{\lambda}^{2} \times L^{\infty}$ into itself and is locally lipschitzean. It is true for the first component since curl maps $L_{\lambda}^{2}$ linearly into itself with norm less than $2 \lambda$. The second component $U \mapsto-\operatorname{curl} E-S^{\lambda} F(M, H)$ maps $L_{\lambda}^{2} \times L_{\lambda}^{2} \times L^{\infty}$ into $L_{\lambda}^{2}$ since

$$
\|F(M, H)\|_{L^{2}} \leq C\left(\|M\|_{L^{\infty}}\right)\|H\|_{L^{2}}
$$

and $S^{\lambda}$ maps $L^{2}$ into $L_{\lambda}^{2}$. Moreover, writing $F(M, H)-F\left(M^{\prime}, H^{\prime}\right)=F(M, H)-$ $F\left(M^{\prime}, H\right)+F\left(M^{\prime}, H-H^{\prime}\right)$ and using (2.4) and the fact that $S^{\lambda}$ is norm one in $L^{2}$, we get

$$
\left\|S^{\lambda} F(M, H)-S^{\lambda} F\left(M^{\prime}, H^{\prime}\right)\right\|_{L^{2}} \leq C(R)\left\|M-M^{\prime}\right\|_{L^{\infty}}+C(R)\left\|H-H^{\prime}\right\|_{L^{2}}
$$


if $\|M\|_{L^{\infty}} \leq R,\left\|M^{\prime}\right\|_{L^{\infty}} \leq R,\|H\|_{L^{2}} \leq R$. The third component belongs to $L^{\infty}$ since inequalities (2.4) and (3.7) imply that

$$
\|F(M, H)\|_{L^{\infty}} \leq C\left(\|M\|_{L^{\infty}}\right)\|H\|_{L^{\infty}} \leq(2 \lambda)^{3 / 2} C\left(\|M\|_{L^{\infty}}\right)\|H\|_{L^{2}} .
$$

Moreover

$$
\begin{gathered}
\left\|F(M, H)-F\left(M^{\prime}, H^{\prime}\right)\right\|_{L^{\infty}} \leq C(R)\left(\left\|M-M^{\prime}\right\|_{L^{\infty}}\|H\|_{L^{\infty}}+\left\|H-H^{\prime}\right\|_{L^{\infty}}\right) \\
\leq(2 \lambda)^{3 / 2}\left(R C(R)\left\|M-M^{\prime}\right\|_{L^{\infty}}+C(R)\left\|H-H^{\prime}\right\|_{L^{2}}\right) .
\end{gathered}
$$

for $\|M\|_{L^{\infty}} \leq R,\left\|M^{\prime}\right\|_{L^{\infty}} \leq R,\|H\|_{L^{2}} \leq R$.

2) The usual theorem for ordinary differential equations in Banach spaces applies to (3.6). There exist $T>0$ and $U^{\lambda} \in C^{1}\left(\left[0, T\left[; L_{\lambda}^{2} \times L_{\lambda}^{2} \times L^{\infty}\right)\right.\right.$ such that $U^{\lambda}$ is the unique maximal solution of (3.6).

3) The identity (3.1) follows from (2.2), like (2.6). Let $R$ be such that $\left\|M_{0}\right\|_{L^{\infty}} \leq R$. Multiplying the first and second equations in (2.10) by $E^{\lambda}$ and $H^{\lambda}$, we get, using (3.1) and (2.4),

$$
\partial_{t}\left\|E^{\lambda}(t)\right\|_{L^{2}}^{2}+\partial_{t}\left\|H^{\lambda}(t)\right\|_{L^{2}}^{2} \leq C(R)\left\|H^{\lambda}(t)\right\|_{L^{2}}^{2},
$$

from which the second estimate (3.2) follows, with $C=C(R)$. This proves that $T=\infty$ as claimed.

4) The first equation in (2.10) implies that $\partial_{t} \operatorname{div} E^{\lambda}=0$. The right hand side of the second equation is $-\partial_{t} S^{\lambda} M^{\lambda}$ and therefore $\partial_{t}\left(\operatorname{div} H^{\lambda}+S^{\lambda} M^{\lambda}\right)=0$. Since the initial conditions satisfy $\operatorname{div} E_{0}=\operatorname{div}\left(H_{0}+M_{0}\right)=0$, one has $\operatorname{div} E_{0}^{\lambda}=$ $\operatorname{div}\left(H_{0}^{\lambda}+S^{\lambda} M_{0}\right)=0$ and (3.3) (3.4) follow. This ends the proof of Proposition 3.1 .

We proceed now with the proof of Theorem 2.6. Because of (3.1), (3.2) the set $\left(U^{\lambda}\right)_{\lambda}$ is weakly relatively compact in $L_{l o c}^{2}\left(\left[0,+\infty\left[; L^{2}\left(\mathbf{R}^{3}\right)\right)\right.\right.$. Extracting a subsequence, we may suppose that the family $\left(U^{\lambda}\right)_{\lambda}$ converges weakly to $U^{\infty}$ in $L_{l o c}^{2}\left(\left[0,+\infty\left[; L^{2}\left(\mathbf{R}^{3}\right)\right)\right.\right.$ thus in $L^{2}\left([0, T] ; L^{2}\left(\mathbf{R}^{3}\right)\right)$, for every $T>0$. The proof aims at showing that $U^{\infty}$ is a global finite energy solution. This involves getting continuity properties of the nonlinear term. A key step in this process is a weighted $L^{2}$ estimate on differences $M^{\lambda}-M^{\mu}$ with a weight that depends on the solution $U^{\infty}$. Plugging

$$
\begin{aligned}
F\left(M^{\lambda}, H^{\lambda}\right)-F\left(M^{\mu}, H^{\mu}\right)= & F\left(M^{\lambda}, H^{\infty}\right)-F\left(M^{\mu}, H^{\infty}\right) \\
& +F\left(M^{\lambda}, H^{\lambda}-H^{\infty}\right)-F\left(M^{\mu}, H^{\mu}-H^{\infty}\right),
\end{aligned}
$$

into the difference of the equations for $M^{\lambda}$ and $M^{\mu}$ in (2.10) and using (2.4) and (3.1) yields the pointwise estimate 


$$
\begin{aligned}
& \frac{1}{2} \partial_{t}\left|M^{\lambda}-M^{\mu}\right|^{2} \leq C(R)\left|H^{\infty}\right|\left|M^{\lambda}-M^{\mu}\right|^{2} \\
& +\left(F\left(M^{\lambda}, H^{\lambda}-H^{\infty}\right)-F\left(M^{\mu}, H^{\mu}-H^{\infty}\right)\right) \cdot\left(M^{\lambda}-M^{\mu}\right),
\end{aligned}
$$

for some $R$ such that $\left|M_{0}(x)\right| \leq R$. The weight $e^{-2 a(t, x)}$ absorbs the first term in the right hand side of $(3.9)$ if $\partial_{t} a(t, x)=C(R)\left|H^{\infty}(t, x)\right|$. The precise choice

$$
a(t, x)=|x|^{2}+\int_{0}^{t} C(R)\left|H^{\infty}(s, x)\right| d s
$$

provides a positive and almost everywhere finite function such that $e^{-2 a(t)} \in$ $L^{p}\left(\mathbf{R}^{3}\right)$ for all $1 \leq p \leq \infty$ which will be used later. With $a$ defined by (3.10), (3.9) yields

$\frac{1}{2} \partial_{t}\left(e^{-2 a}\left|M^{\lambda}-M^{\mu}\right|^{2}\right) \leq e^{-2 a}\left(F\left(M^{\lambda}, H^{\lambda}-H^{\infty}\right)-F\left(M^{\mu}, H^{\mu}-H^{\infty}\right)\right) \cdot\left(M^{\lambda}-M^{\mu}\right)$.

Proposition 3.2 There is a constant $C(R, T)$ such that for all $\delta>0$ there exist

$N(\delta)$ such that for all $\lambda \geq N(\delta)$ and $\mu \geq N(\delta)$ and all $t \in[0, T]$,

$$
\left\|e^{-a(t)}\left(M^{\lambda}(t)-M^{\mu}(t)\right)\right\|_{L^{2}}^{2} \leq C\left(\delta+\int_{0}^{t}\left\|e^{-a(s)}\left(M^{\lambda}(s)-M^{\infty}(s)\right)\right\|_{L^{2}}^{2} d s\right) .
$$

We postpone the proof of Proposition 3.2 until next section and finish the proof of Theorem 2.6. We fix $T>0$ and prove that $U^{\infty}$ is a finite energy solution on $\Omega_{T}$.

1) We first show that for all $t \in[0, T], M^{\mu}(t)$ converges weakly in $L^{2}$ to $M^{\infty}(t)$. This result being independent of Proposition 3.2 can be used for its proof.

The family $M^{\lambda}$ is bounded in $L^{\infty}$ and $\left(E^{\lambda}, H^{\lambda}\right)$ is bounded in $C^{0}\left([0, T] ; L^{2}\right)$, as a consequence of (3.1) (3.2). Therefore, the third equation in (2.10) and the estimate (2.4) imply that there is a constant $K$ such that for all $\mu$ and $t \leq t^{\prime}$ in $[0, T]$

$$
\left\|M^{\mu}(t)-M^{\mu}\left(t^{\prime}\right)\right\|_{L^{2}} \leq K\left|t-t^{\prime}\right|
$$

Thus $\left\{M^{\mu}\right\}_{\mu}$ is equicontinuous in time with value in $L^{2}$. Ascoli's Theorem implies that for all test function $\varphi$, the family of functions $t \mapsto \int M^{\mu}(t, x) \varphi(x) d x$, which is bounded and equicontinuous, has subsequences which converge in $C^{0}([0, T])$. Since $U^{\lambda} \rightarrow U^{\infty}$ weakly, it follows that $\int \psi(t) \varphi(x) M^{\mu}(t, x) d t d x$ converges to $\int \psi(t) \varphi(x) M^{\infty}(t, x) d t d x$. This shows that $\int M^{\mu}(t, x) \varphi(x) d x$ converges to $\int \varphi(x)$ 
$M^{\infty}(t, x) d x$ uniformly in $t$. Therefore, for all $t \in[0, T], M^{\mu}(t)$ converges weakly in $L^{2}$ to $M^{\infty}(t)$.

As a consequence, for all $t \in[0, T]$, we have

$$
\left\|e^{-a(t)}\left(M^{\lambda}-M^{\infty}\right)(t)\right\|_{L^{2}}^{2} \leq C \liminf _{\mu}\left\|e^{-a(t)}\left(M^{\lambda}-M^{\mu}\right)(t)\right\|_{L^{2}}^{2} .
$$

Letting $\mu$ tends to infinity in (3.12) implies that that for all $\lambda \geq N(\delta)$

$$
\left\|e^{-a(t)}\left(M^{\lambda}-M^{\infty}\right)(t)\right\|_{L^{2}}^{2} \leq C\left(\delta+\int_{0}^{t}\left\|e^{-a(s)}\left(M^{\lambda}-M^{\infty}\right)(s)\right\|_{L^{2}}^{2} d s\right) .
$$

Gronwall's Lemma and (3.13) imply that $M^{\lambda}$ converge to $M^{\infty}$ in $L^{2}\left(\Omega_{T}, e^{-a(t, x)}\right.$ $d t d x)$ ). Since $a$ is finite almost everywhere, $e^{-a} \neq 0$ almost everywhere. Thus, from any subsequence of $M^{\lambda}$ we can extract a subsequence converging pointwise almost everywhere and thus in $L^{2}\left(\Omega_{T}, d t d x\right)$ thanks to the pointwise estimate (3.1) and Lebesgue's Dominated Convergence Theorem. The limit is $M^{\infty}$, and the convergence holds for the full sequence. Thus $t \mapsto\left\|M^{\lambda}(t)-M^{\mu}(t)\right\|_{L^{2}}$ converges to 0 in $L^{2}([0, T])$. Since the sequence $M^{\lambda}$ is equicontinuous in $t$ with value in $L^{2}$ the above convergence holds in $C^{0}([0, T])$. Thus $M^{\lambda} \rightarrow M^{\infty}$ in $C^{0}\left([0, T] ; L^{2}\right)$.

2) We show that $S^{\lambda}\left(F\left(M^{\lambda}, H^{\lambda}\right)\right)$ converges in $L^{1}\left([0, T] ; L^{2}\right)$ to $F\left(M^{\infty}, H^{\infty}\right)$ and $\left(E^{\lambda}, H^{\lambda}\right)$ converges to $\left(E^{\infty}, H^{\infty}\right)$ in $C^{0}\left([0, T] ; L^{2} \times L^{2}\right)$. The Maxwell system for the difference $\left(E^{\lambda}-E^{\infty}, H^{\lambda}-H^{\infty}\right)$ involves the source term $\delta F=$ $S^{\lambda}\left(F\left(M^{\lambda}, H^{\lambda}\right)\right)-F\left(M^{\infty}, H^{\infty}\right)$ which we write

$$
\delta F=S^{\lambda}\left(A^{\lambda}\right)+S^{\lambda}\left(B^{\lambda}\right)+\left(I-S^{\lambda}\right)\left(F\left(M^{\infty}, H^{\infty}\right)\right) .
$$

with

$$
A^{\lambda}=F\left(M^{\lambda}, H^{\lambda}-H^{\infty}\right), \quad B^{\lambda}=F\left(M^{\lambda}, H^{\infty}\right)-F\left(M^{\infty}, H^{\infty}\right) .
$$

The $L^{2}$ estimate for the Maxwell system implies that

$$
\|(\delta E(t), \delta H(t))\|_{L^{2}} \leq C\left(\|(\delta E(0), \delta H(0))\|_{L^{2}}+\int_{0}^{t}\|\delta F(s)\|_{L^{2}}, d s\right) .
$$

We need to estimate the $L^{1}\left([0, T] ; L^{2}\right)$ norm of the 3 terms of the decomposition (3.14). The uniform estimate of $M^{\lambda}$ and (2.4) yield

$$
\int_{0}^{t}\left\|S^{\lambda}\left(A^{\lambda}\right)(s)\right\|_{L^{2}} d s \leq C \int_{0}^{t}\|\delta H(s)\|_{L^{2}} d s .
$$

Using (2.4), one has the pointwise estimate

$$
\left|B^{\lambda}(s, x)\right| \leq C(R)\left|M^{\lambda}(s, x)-M^{\infty}(s, x)\right|\left|H^{\infty}(s, x)\right| \leq 2 R C(R)\left|H^{\infty}(s, x)\right|,
$$


which proves that $B^{\lambda}$ is dominated by a function that belongs to $L^{1}\left([0, T] ; L^{2}\right)$. Moreover, since $M^{\lambda}$ converges to $M^{\infty}$ in $L^{2}\left(\Omega_{T}\right)$, (3.17) also implies that $B^{\lambda}(s, x) \rightarrow 0$ in $L^{1}\left(\Omega_{T}\right)$. Lebesgue's Theorem implies that $B^{\lambda}(s, x) \rightarrow 0$ in $L^{2}\left(\Omega_{T}\right)$ thus in $L^{1}\left([0, T] ; L^{2}\right)$. The same result holds for the third term since $S^{\lambda} \rightarrow I$ pointwise in $L^{2}$. Since $(\delta E(0), \delta H(0))$ converges to 0 in $L^{2}$, estimate $(3.15)$ and Gronwall's Lemma imply that $(\delta E(t), \delta H(t))$ converges to 0 in $L^{2}$, uniformly for $t \in[0, T]$.

3) We have proved that $U^{\lambda}$ converges to $U^{\infty}$ in $C^{0}\left([0, T] ; L^{2}\right)$ for all $T>$ 0. Thus $F\left(M^{\lambda}, H^{\lambda}\right)$ and $S^{\lambda}\left(F\left(M^{\lambda}, H^{\lambda}\right)\right.$ converge to $F\left(M^{\infty}, H^{\infty}\right)$ and $U^{\infty}=$ $\left(E^{\infty}, H^{\infty}, M^{\infty}\right)$ is a solution to $(1.2)$ in the distribution sense satisfying $U^{\infty}(0)=$ $U_{0}$. The estimates (3.1) imply that $M^{\infty} \in L^{\infty}\left(\Omega_{\infty}\right)$. Equalities

$$
\operatorname{div} E^{\infty}=\operatorname{div}\left(H^{\infty}+M^{\infty}\right)=0
$$

follow from (3.3), (3.4) letting $\lambda \rightarrow \infty$. This achieves the proof that $U^{\infty}$ is a finite energy solution of with initial data $U_{0}$.

The proof of Theorem 2.6 is now complete. It implies the first part of Theorem 2.5. To end the proof of Theorem 2.5, consider a bounded sequence in $\mathbf{L}_{\mathbf{0}}$ of Cauchy data $U_{0}^{n}$, such that $U_{0}^{n}$ converges to $U_{0}^{\infty}$ in $L^{2}$. Denote by $U^{n}$ a finite energy solution such that $U_{\mid t=0}^{n}=U_{0}^{n}$. We need to show there exists a subsequence, still denoted by $U^{n}$, which converges strongly to a finite energy solution $U^{\infty}$ with initial data $U_{\mid t=0}^{\infty}=U_{0}^{\infty}$. The proof is quite parallel to the proof of Theorem 2.6. The a-priori estimates (2.5) (2.6) show that $U^{n}$ is bounded in $C^{0}\left(\left[0,+\infty\left[; L^{2}\right)\right.\right.$ and $M^{n}$ is bounded in $L^{\infty}\left(\Omega_{\infty}\right)$. Therefore, extracting a subsequence, one can assume that $U^{n}$ converges weakly to $U^{\infty}$ in $L^{2}\left(\Omega_{T}\right)$ for all $T>0$. With $a$ given by (3.10), the inequality (3.11) holds for $U^{n}$ and $U^{n^{\prime}}$ and (3.12) is to be replaced by

$$
\begin{gathered}
\left\|e^{-a(t)}\left(M^{n}(t)-M^{n^{\prime}}(t)\right)\right\|_{L^{2}}^{2} \leq\left\|e^{-a(0)}\left(M_{0}^{n}-M_{0}^{n^{\prime}}\right)\right\|_{L^{2}}^{2} \\
+C\left(\delta+\int_{0}^{t}\left\|e^{-a(s)}\left(M^{n}(s)-M^{\infty}(s)\right)\right\|_{L^{2}}^{2} d s\right) .
\end{gathered}
$$

Using this estimate and the equicontinuity of $M^{n}(t)$, one deduces the strong convergence $M^{n} \rightarrow M^{\infty}$ in $C^{0}\left([0, T] ; L^{2}\right)$ for all $T>0$ as before. The strong convergence $\left(E^{n}, H^{n}\right) \rightarrow\left(E^{\infty}, H^{\infty}\right)$ follows from the energy estimate (3.15), with the simplification that there is no $S^{\lambda}$ in the analogues of (3.14-16). The strong convergence imply that $F\left(M^{n}, H^{n}\right) \rightarrow F\left(M^{\infty}, H^{\infty}\right)$ which proves that $U^{\infty}$ is a finite energy solution with initial data $U_{0}$.

\section{Proof of Proposition 3.2.}

Note that $M^{\lambda}$ and $M^{\mu}$ have the same initial data $M_{0}$. Integrating (3.11) in $t, x$ yields 


$$
\begin{aligned}
& \left\|e^{-a(t)}\left(M^{\lambda}(t)-M^{\mu}(t)\right)\right\|_{L^{2}}^{2} \leq \\
& \quad 2 \int_{0}^{t} \int e^{-2 a(s)}\left(F\left(M^{\lambda}(s), H^{\lambda}(s)-H^{\infty}(s)\right)\right) \cdot\left(M^{\lambda}(s)-M^{\mu}(s)\right) d s d x \\
& \left.\quad-2 \int_{0}^{t} \int e^{-2 a(s)}\left(F\left(M^{\mu}(s), H^{\mu}(s)-H^{\infty}(s)\right)\right) \cdot\left(M^{\lambda}(s)-M^{\mu}(s)\right) d s d x \mathrm{IV} .1\right)
\end{aligned}
$$

We then write each term $F\left(M^{\rho}, H^{\rho}-H^{\infty}\right)$ in the right hand side of (4.1), where $\rho$ stands for $\lambda$ or $\mu$ as the sum of two terms, using the linearity of $F$ with respect to $H$ and the decomposition (2.12), $H^{\rho}=H_{\perp}^{\rho}+H_{\|}^{\rho}$ and $H^{\infty}=H_{\perp}^{\infty}+H_{\|}^{\infty}$. The equation (3.4) implies that $H_{\|}^{\rho}=-S^{\rho} P_{\|} M^{\rho}$. Taking weak limits in (3.4) implies that $\operatorname{div}\left(H^{\infty}+M^{\infty}\right)=0$ and thus $H_{\|}^{\infty}=-P_{\|} M^{\infty}$. Accordingly, the two terms in the right hand side of (4.1) are equal to

$$
\begin{gathered}
\int_{0}^{t} \int e^{-2 a(s)}\left(F\left(M^{\rho}(s), H_{\perp}^{\rho}(s)-H_{\perp}^{\infty}(s)\right) \cdot\left(M^{\lambda}(s)-M^{\mu}(s)\right) d x d s\right. \\
-\int_{0}^{t} \int e^{-2 a(s)}\left(F\left(M^{\rho}(s), S^{\rho} P_{\|}\left(M^{\rho}(s)-M^{\infty}(s)\right)\right) \cdot\left(M^{\lambda}(s)-M^{\mu}(s)\right) d x d s\right. \\
\int_{0}^{t} \int e^{-2 a(s)}\left(F\left(M^{\rho}(s),\left(I-S^{\rho}\right) P_{\|}\left(M^{\infty}(s)\right)\right)\right) \cdot\left(M^{\lambda}(s)-M^{\mu}(s)\right) d x d s .
\end{gathered}
$$

The linearity of $F$ with respect to $H$ implies that (4.3) is the sum of

$-\int_{0}^{t} \int\left(F\left(M^{\rho}(s), S^{\rho} P_{\|}\left(e^{-a(s)}\left(M^{\rho}(s)-M^{\infty}(s)\right)\right)\right)\right) \cdot e^{-a(s)}\left(M^{\lambda}(s)-M^{\mu}(s)\right) d x d s$

and

$-\int_{0}^{t} \int\left(F\left(M^{\rho}(s),\left[e^{-a(s)}, S^{\rho} P_{\|}\right]\left(M^{\rho}(s)-M^{\infty}(s)\right)\right)\right) \cdot e^{-a(s)}\left(M^{\lambda}(s)-M^{\mu}(s)\right) d x d s$

where $[A, B]=A B-B A$. The main step in the proof of Proposition 3.2 is to show that (4.2) and (4.6) are small when $\lambda$ and $\mu$ are large as asserted by the following two propositions to be proved later.

Proposition 4.1 For all $\delta>0$ there exists $N(\delta)>0$ such that for all $\lambda, \mu \geq N(\delta)$ and for all $0 \leq t \leq T$,

$$
\mid \int_{0}^{t} \int e^{-2 a(s)}\left(F\left(M^{\rho}(s), H_{\perp}^{\rho}(s)-H_{\perp}^{\infty}(s)\right) \cdot\left(M^{\lambda}(s)-M^{\mu}(s)\right) d s d x \mid \leq \delta .\right.
$$


Proposition 4.2 For all $\delta>0$ there exists $N(T, \delta)$ such that for all $\lambda, \mu \geq N$ and all $0 \leq t \leq T$,

$$
\left|\int_{0}^{t} \int F\left(M^{\rho}(t),\left[e^{-a(t)}, S^{\rho} P_{\|}\right]\left(M^{\infty}(t)-M^{\rho}(t)\right)\right) \cdot\left(M^{\lambda}(t)-M^{\mu}(t)\right) d x d t\right| \leq \delta .
$$

Since $P_{\|} M^{\infty} \in L^{2}\left(\Omega_{T}\right),\left(I-S^{\rho}\right) P_{\|} M^{\infty}$ converges to 0 in $L^{2}$. Together with the uniform bounds for $M^{\lambda}$, this implies that

$$
\left.\mid \int_{0}^{t} \int e^{-2 a(s)}\left(F\left(M^{\rho}(s), S^{\rho}\left(I-S^{\rho}\right) P_{\|} M^{\infty}(s)\right)\right)\right) \cdot\left(M^{\lambda}(s)-M^{\mu}(s)\right) d x d s \mid \leq \delta
$$

when $\lambda$ and $\mu$ are large enough. by

Using (2.4) and that $S^{\rho} P_{\|}$is bounded in $L^{2}$, the term in (4.5) is estimated

$$
\frac{C(R)}{2}\left(\int_{0}^{t}\left\|e^{-a(s)}\left(M^{\rho}(s)-M^{\infty}(s)\right)\right\|_{L^{2}}^{2} d s+\int_{0}^{t}\left\|e^{-a(s)}\left(M^{\lambda}(s)-M^{\mu}(s)\right)\right\|_{L^{2}}^{2} d s\right) .
$$

When $\rho=\mu$, we substitute $\left(M^{\mu}-M^{\lambda}\right)+\left(M^{\lambda}-M^{\infty}\right)$ in place of $M^{\rho}-M^{\infty}$. Therefore, the sum of the two terms (4.10) is less than or equal to

$$
2 C(R)\left(\int_{0}^{t}\left\|e^{-a(s)}\left(M^{\lambda}(s)-M^{\infty}(s)\right)\right\|_{L^{2}}^{2} d s+\int_{0}^{t}\left\|e^{-a(s)}\left(M^{\lambda}(s)-M^{\mu}(s)\right)\right\|_{L^{2}}^{2} d s\right) .
$$

The estimates above show that for all $\delta>0$, there is $N(\delta, T)$ such that for all $\lambda \geq N(\delta, T), \mu \geq N(\delta, T)$ and $t \in[0, T]$, the right hand side of (4.1) is less than or equal to $\delta$ plus twice the term in (4.11). Using Gronwall's Lemma, the estimate (3.12) follows

Proof of Proposition 4.1. The linearity of $F$ yields

$$
F\left(M^{\rho}, H_{\perp}^{\rho}-H_{\perp}^{\infty}\right) \cdot\left(M^{\lambda}-M^{\mu}\right)=G\left(M^{\lambda}, M^{\mu}\right) \cdot\left(H_{\perp}^{\rho}-H_{\perp}^{\infty}\right)
$$

where $G$ is lipschitzean so that the function $A^{\lambda, \mu}(s, x)$ to be integrated in (4.7) reads $A^{\lambda, \mu}=e^{-2 a} G\left(M^{\lambda}, M^{\mu}\right) \cdot\left(H_{\perp}^{\rho}-H_{\perp}^{\infty}\right)$ and is the product of $e^{-2 a} G\left(M^{\lambda}, M^{\mu}\right)$ by $H_{\perp}-H_{\perp}^{\infty}$. We now study the regularity properties of each factor of this product. The Lipschitz property of $G,(3.1), M_{0} \in L^{2} \cap L^{\infty}$ and (3.10) imply that

$$
\left\|G\left(M^{\lambda}, M^{\mu}\right)\right\|_{C^{0}\left([0, T] ; L^{2}\right)} \leq C, \quad\left\|\partial_{t} G\left(M^{\lambda}, M^{\mu}\right)\right\|_{L^{2}\left(\Omega_{T}\right)} \leq C .
$$

Here we have used that the Lipschitz regularity of $G$ is sufficient to differentiate $G\left(M^{\lambda}, M^{\mu}\right)$ with respect to $t$. From (2.10), we get

$$
\square H_{\perp}^{\rho}=-\partial_{t}^{2} M^{\rho}=-\partial_{t} P_{\perp} S^{\rho} F\left(M^{\rho}, H^{\rho}\right) .
$$


Thus $\square H_{\perp}^{\rho}$ is bounded in $H^{-1}\left(\Omega_{T}\right)$ and $\square H_{\perp}^{\infty} \in H^{-1}\left(\Omega_{T}\right)$. Moreover,

$$
\left\|H_{\perp}-H_{\perp}^{\infty}\right\|_{C^{0}\left([0, T] ; L^{2}\right)} \leq C, \quad\left\|\square\left(\left(H_{\perp}-H_{\perp}^{\infty}\right)\right)\right\|_{H^{-1}\left(\Omega_{T}\right)} \leq C .
$$

In (4.12), (4.13), $C$ is a constant which only depends on the Cauchy data and $\varphi$. The a-priori estimates (4.13) implies that the microlocal defect measures of $H^{\rho}$ $H^{\infty}$ is contained in the characteristic variety of $\square$, that is $\mathcal{C}_{\square}=\left\{\tau^{2}=|\xi|^{2}\right\} \backslash\{0\}$. Similarly, the microlocal defect measures of $G\left(M^{\lambda}, M^{\mu}\right)$ is contained in $\mathcal{C}_{\partial_{t}}=\{\tau=$ $0\} \backslash\{0\}$. Since $0 \notin \mathcal{C}_{\square}+\mathcal{C}_{\partial_{t}}$, this implies that the product $G\left(M^{\lambda}, G^{\mu}\right)\left(H^{\rho}-H^{\infty}\right)$ tends to 0 in $L_{l o c}^{1}\left(\Omega_{T}\right)$, see [Ta], [Gé]. This result can also be obtained as a consequence of theorems of multiplications of distributions with microlocal additional smoothness.

In addition, (4.12) and (4.13) show that $G\left(M^{\lambda}, G^{\mu}\right)\left(H^{\rho}-H^{\infty}\right)$ is bounded in $C^{0}\left([0, T], L^{1}\right)$. Since the weight $e^{-2 a}$ tends to zero as $|x| \rightarrow \infty$, we conclude that $G\left(M^{\lambda}, G^{\mu}\right)\left(H^{\rho}-H^{\infty}\right)$ tends to 0 in $L^{1}\left(\Omega_{T}\right)$ and Proposition 4.1 is proved.

The proof of Proposition 4.2 relies on the following lemma.

Lemma 4.3 For all $0 \leq t \leq T$,

$$
\lim _{\lambda \rightarrow \infty}\left\|\left[e^{-a(t)}, S^{\rho} P_{\|}\right]\left(M^{\infty}(t)-M^{\rho}(t)\right)\right\|_{L^{2}}=0
$$

Proof. Fix $t \in[0, T]$. Write $\left[e^{-a(t)}, S^{\rho} P_{\|}\right]=\left[e^{-a(t)}-b, S^{\rho} P_{\|}\right]+\left[b, S^{\rho} P_{\|}\right]$, where $b \in C_{0}^{\infty}$. Since $S^{\rho} P_{\|}$define a bonded family of continuous operators on $L^{4}$ and $L^{2}$, there exists a constant $C$ such that

$$
\left\|\left[e^{-a(t)}-b, S^{\rho} P_{\|}\right]\left(M^{\infty}(t)-M^{\rho}(t)\right)\right\|_{L^{2}} \leq C\left\|e^{-a(t)}-b\right\|_{L^{4}}\left\|M^{\infty}(t)-M^{\rho}(t)\right\|_{L^{4}} .
$$

Fix $\delta>0$. Estimate (3.1) implies that the sequence $M^{\infty}(t)-M^{\rho}(t)$ is bounded in $L^{\infty}$ and $L^{2}$ hence in $L^{4}$. We choose $b \in C_{0}^{\infty}\left(\mathbf{R}^{3}\right)$ such that $\left\|e^{-a(t)}-b\right\|_{L^{4}}$ is so small that the left hand side of (4.15) is less than $\delta / 3$ for $\rho \geq 1$.

Choose now $\psi_{1}$ and $\psi_{2}$ in $C_{0}^{\infty}$ such that $b \psi_{1}=b$ and $\psi_{1} \psi_{2}=\psi_{1}$. The commutators $\left[b, S^{\rho} P_{\|}\right]$form a bounded family of pseudodifferential operators of degree -1 . Since $\left(M^{\rho}-M^{\infty}\right)(t)$ converges weakly to zero in $L^{2}$, as remarked in the first step of the proof of Theorem 2.6, this implies that $\psi_{2}\left[b, S^{\rho} P_{\|}\right]\left(M^{\rho}(t)-M^{\infty}(t)\right.$ converges strongly to 0 in $L^{2}$. On the other hand $b\left(M^{\rho}(t)-M^{\infty}(t)\right.$ converge strongly to 0 in $H^{-1}$ and $\left(1-\psi_{2}\right)\left[b, S^{\rho} P_{\|}\right]=\left(1-\psi_{2}\right) S^{\rho} P_{\|} \psi_{1} b\left(M^{\rho}(t)-M^{\infty}(t)\right.$ converge strongly to 0 in $L^{2}$ since $\left(1-\psi_{2}\right) S^{\rho} P_{\|} \psi_{1}$ is a bounded family of operators of degree -1 .

Remark. Consider $m(x) \in L^{\infty}$ and $p(D)$ of order 0 . Then $[m, p(D)]$ is not, in general, a compact operator on $L^{2}$. What Lemma 4.3 shows is that this commutator is compact when restricted to bounded subsets of $L^{p} \cap L^{2}$ and when $m$ belongs to 
$L^{q}$ with $1 / p+1 / q=1 / 2, p>2$. This is the main reason why we introduced the term $|x|^{2}$ in the definition (3.10) of the weight $a$.

Proof of Proposition 4.2. From (3.1), (2.4) and Cauchy-Schwarz inequality, it follows that the family $t \mapsto F\left(M^{\rho}(t),\left[e^{-a(t)}, S^{\rho} P_{\|}\right]\left(M^{\infty}(t)-M^{\rho}(t)\right)\right) \cdot\left(M^{\lambda}(t)-\right.$ $\left.M^{\mu}(t)\right)$ is bounded in $L^{\infty}\left([0, T] ; L^{1}\right)$. From Lemma 4.3 it also follows that $t \mapsto$ $\left\|F\left(M^{\rho}(t),\left[e^{-a(t)}, S^{\rho} P_{\|}\right]\left(M^{\infty}(t)-M^{\rho}(t)\right)\right) \cdot\left(M^{\lambda}(t)-M^{\mu}(t)\right)\right\|_{1}$ converges pointwise to 0. Lebesgue's Dominated Convergence Theorem implies that

$$
\int_{0}^{T} \int\left|F\left(M^{\rho}(t),\left[e^{-a(t)}, S^{\rho} P_{\|}\right]\left(M^{\infty}(t)-M^{\rho}(t)\right)\right) \cdot\left(M^{\lambda}(t)-M^{\mu}(t)\right)\right| d x d t \rightarrow 0
$$

as $\lambda, \mu \rightarrow \infty$, thus achieving the proof of Proposition 4.3.

\section{Curl estimates for the electromagnetic field}

In this section we prove Theorem 2.7. We first consider the case $\mu<1$ and next use the $H^{1 / 2}$ estimate to prove the $H^{1}$ regularity. Consider a finite energy solution $U=(E, H, M)$ on $\Omega_{\infty}$. We show that if the initial data $E_{0}$ and $H_{0}$ have curl in the Sobolev space $H^{\mu-1}$ then the same property holds for all time. We use the projectors $P_{\|}$and $P_{\perp}$ introduced in (2.12). Apply $P_{\perp}$ to the first two equations in (1.2). Using (2.13) and the identity $E=E_{\perp}, \operatorname{curl} H=\operatorname{curl} H_{\perp}$, yields

$$
\left\{\begin{array}{l}
\partial_{t} E_{\perp}-\operatorname{curl} H_{\perp}=0 \\
\partial_{t} H_{\perp}+\operatorname{curl} E_{\perp}=P_{\perp}\left(A H_{\perp}\right)+P_{\perp} g
\end{array}\right.
$$

where $A$ is such that $F(M, H)=-A H$ and $g:=A M_{\|}$. We consider (5.1) as a linear system for $u=\left(E_{\perp}, H_{\perp}\right)$,

$$
L u:=\partial_{t} u+\Lambda\left(\partial_{x}\right) u=P(a u)+P g,
$$

with a given coefficient $a$ and a given source term $P g . P=P\left(D_{x}\right)$ is a Fourier multiplier with $P(\xi)$ a projector in $\mathbf{C}^{6}$ which is $C^{\infty}$ and homogeneous of degree 0. $\Lambda(\xi)$ commutes to $P(\xi)$ and $\Lambda(\xi) P(\xi)$ has eigenvalues of constant multiplicity $\pm|\xi|$.

We know that $M \in L^{\infty}\left(\Omega_{\infty}\right)$ and $M$ and $H$ are continuous and bounded in time with values in $L^{2}$. Therefore $\partial_{t} M=F(M, H) \in L^{\infty}\left(\left[0, \infty\left[; L^{2}\right) \cap\right.\right.$ $C^{0}\left(\left[0,+\infty\left[; L^{p}\right)\right.\right.$ for all $p<\infty$. The same regularity holds for any Lipschitz function of $M$ and thus $a$ and $g$ satisfy

$$
\begin{aligned}
& a \in L^{\infty}\left(\Omega_{\infty}\right) \cap C^{0}\left(\left[0,+\infty\left[; L^{2}\right), \quad \partial_{t} a \in L^{\infty}\left(\left[0,+\infty\left[; L^{2}\right),\right.\right.\right.\right. \\
& g \in C^{0}\left(\left[0,+\infty\left[; L^{\sigma}\right) \quad \text { for all } \sigma<\infty\right.\right. \text {, } \\
& \partial_{t} g \in L^{\infty}\left(\left[0,+\infty\left[; L^{q}\right) \text { for all } q<2\right.\right. \text {. }
\end{aligned}
$$

Note that $L$ is symmetric hyperbolic. Therefore the Cauchy problem for (5.2), with initial data in $u_{0} \in L^{2}$ such that $P u_{0}=u_{0}$ has a unique solution $u \in$ $C^{0}\left(\left[0,+\infty\left[; L^{2}\right)\right.\right.$ which satisfies $P u=u$. When $\mu<1$, Theorem 2.7 follows from the next proposition. 
Proposition 5.1 Suppose that $a$ and $g$ satisfy (5.3) (5.4), $\mu \in] 0,1\left[\right.$ and $u_{0} \in H^{\mu}$ satisfies $P u_{0}=u_{0}$. Then the unique solution $u$ to (5.2) with initial data $u_{0}$ belongs to $C^{0}\left(\left[0,+\infty\left[; H^{\mu}\right)\right.\right.$.

For $T>0$, introduce the space

$$
Y^{\mu}(T)=C^{0}\left([0, T], H^{\mu}\right) \cap C^{1}\left([0, T] ; H^{\mu-1}\right) \cap L^{r}\left([0, T] ; B_{p, 2}^{0}\right)
$$

where $2 / p=1-\mu, 2 / r=\mu$ and $B_{p, p^{\prime}}^{s}$ denotes the scale of Besov spaces in $\mathbf{R}^{3}$ (the definition is recalled below). Introduce next $Z^{\mu}(T)$, the space of functions $f$ on $\Omega_{T}$ which admits the decomposition $f=f_{1}+f_{2}$ with

$$
f_{1} \in L^{1}\left([0, T], H^{\mu}\right) \cap C^{0}\left([0, T] ; L^{2}\right),
$$

and

$$
\left\{\begin{array}{c}
f_{2} \in C^{0}\left([0, T] ; L^{2}\right) \cap L^{r}\left([0, T] ; B_{p, 2}^{-1}\right), \\
\partial_{t} f_{2} \in L^{1}\left([0, T], H^{\mu-1}\right)+L^{s}\left([0, T] ; B_{q, 2}^{0}\right)
\end{array}\right.
$$

with $2 / q=2-\mu$ and $2 / s=1+\mu$. These spaces are equipped with the obvious norms. The main step in the proof of Proposition 5.1 is to prove a local existence theorem in $Y^{\mu}(T)$, with a control on $T$. This relies on two estimates.

Lemma 5.2 For $T \in] 0,1], f \in Z^{\mu}(T)$ and $u_{0} \in H^{\mu}$ such that $P u_{0}=u_{0}$, the solution $u$ of

$$
L u=P f, \quad u_{t=0}=u_{0}
$$

belongs to $Y^{\mu}(T)$ and satisfies

$$
\begin{aligned}
\|u\|_{C^{0}\left([0, T] ; L^{2}\right)} & \leq\left\|u_{0}\right\|_{L^{2}}+2 T\|f\|_{C^{0}\left([0, T] ; L^{2}\right)}, \\
\|u\|_{Y^{\mu}(T)} & \leq C\left(\left\|u_{0}\right\|_{H^{\mu}}+\|f\|_{Z^{\mu}(T)}\right),
\end{aligned}
$$

where $C$ only depends on $\mu$.

Lemma 5.3 There is a constant $C$ which only depends on $\|a\|_{L^{\infty}},\left\|\partial_{t} a\right\|_{L^{\infty}\left(L^{2}\right)}$ and $\mu$, such, that for all $T \in] 0,1]$ and $u \in Y^{\mu}(T)$, the product au belongs to $Z^{\mu}$ and

$$
\|a u\|_{Z^{\mu}(T)} \leq C T^{\mu / 2}\left\|u_{0}\right\|_{H^{\mu}}+C\|u\|_{C^{0}\left([0, T] ; L^{2}\right)} .
$$

Let $K$ denote the mapping $w \mapsto u$, where $u$ solves (5.8) with $f=a u$ and $u_{0}=0$. The estimates in the two lemmas above show that there is $T_{1}$, which only depends on the norms of $a$, such that $K^{2}$ is a contraction in $Y^{\mu}(T)$ if $T \leq T_{1}$. The estimates (5.4) for $g$ and the embedding $L^{p} \subset B_{p, 2}^{-1}$ and $L^{q} \subset B_{q, 2}^{0}$ for $q \leq 2$ (see $[\operatorname{Tr}]$ ), show that $g \in C^{0}\left(L^{2}\right), g \in L^{r}\left(B_{p, 2}^{-1}\right)$ and $\partial_{t} g \in L^{s}\left(B_{q, 2}^{0}\right)$. Therefore 
$g \in Z^{\mu}(T)$ for all $T$. Thus, the problem (5.2) with initial data $u_{0} \in H^{\mu}$ such that $P u_{0}=u_{0}$ has a solution $u \in Y^{\mu}\left(T_{1}\right)$, which satisfies $P u=u$ and

$$
\left\|u\left(T_{1}\right)\right\|_{H^{\mu}} \leq C\left(\left\|u_{0}\right\|_{H^{\mu}}+\|g\|_{Z^{\mu}\left(T_{1}\right)}\right) .
$$

Since $T_{1}$ only depends on the norms of $a$, the solution can be continued to $2 T_{1}$ and, by induction, to all time. So, to finish the proof of Proposition 5.1, it remains to prove the two lemmas above.

Proof of Lemma 5.2. The linear problem (5.8) has a unique solution which is smooth when the data are smooth. Thus it is sufficient to prove the estimates (5.9) and (5.10) for smooth solutions. The first one is the standard energy estimate in $L^{2}$ for symmetric hyperbolic systems. The main ingredient to prove (5.10) are Strichartz estimates.

1) First we recall the definition of Besov spaces. Introduce $\varphi \in C_{0}^{\infty}\left(\mathbf{R}^{3}\right)$, $0 \leq \varphi \leq 1$, supported in $\{|\xi| \leq 2\}$ and equal to 1 on $\{|\xi| \leq 1\}$. Introduce next

$$
\left\{\begin{array}{cc}
\varphi_{k}(\xi):=\varphi\left(2^{-k} \xi\right) & \text { for } k \geq 0 \\
\psi_{k}(\xi):=\varphi\left(2^{-k} \xi\right)-\varphi\left(2^{-k+1} \xi\right) & \text { for } k \in \mathbf{Z} .
\end{array}\right.
$$

Denote by $S_{k}$ [resp $\Delta_{k}$ ] the Fourier multipliers with symbols $\varphi_{k}$ [resp $\left.\psi_{k}\right]$. Recall that $B_{p, 2}^{s}$ is the space of temperate distributions $u$ such that

$$
\left\|S_{0} u\right\|_{L^{p}}^{2}+\sum_{k=1}^{+\infty} 2^{-2 k s}\left\|\Delta_{k} u\right\|_{L^{p}}^{2}<\infty
$$

Also recall that $H^{\mu}=B_{2,2}^{\mu}([\operatorname{Tr}])$. The homogeneous spaces $\dot{B}_{p, 2}^{s}$ have a similar definition with

$$
\sum_{k=-\infty}^{+\infty} 2^{-2 k s}\left\|\Delta_{k} u\right\|_{L^{p}}^{2}<\infty
$$

in place of (5.14).

2) Introduce the groups of operators $G_{ \pm}(t)$ of Fourier multipliers $e^{ \pm i t|\xi|}$. Since the eigenvalues of $\Lambda(\xi) P(\xi)$ are $\pm|\xi|$ and have constant multiplicity, the fundamental solution of $L$, for data in the kernel of $I-P$, is

$$
G_{+}(t) P_{+}+G_{-}(t) P_{-}
$$

where $P_{ \pm}$are Fourier multipliers with smooth symbols $P_{ \pm}(\xi)$ which are orthogonal projectors, with $P=P_{+}+P_{-}$. The operators $P_{ \pm}$act in $L^{\sigma}$ and in $B_{\sigma, 2}^{s}$ for all $s$ and all $\sigma \in] 1,+\infty\left[\right.$. For $G_{ \pm}$, we use the Strichartz estimates proved in [GV] (see also [LS]). For $v_{0} \in \dot{H}^{\mu}$ and $f \in L^{s_{1}}\left([0, T], \dot{B}_{q_{1}, 2}^{\sigma}\right), v(t)=G_{ \pm}(t) v_{0}$ and $w(t)=$ $\int_{0}^{t} G_{ \pm}\left(t-t^{\prime}\right) f\left(t^{\prime}\right) d t^{\prime}$ belong to $L^{r_{1}}\left([0, T], \dot{B}_{p_{1}, 2}^{\rho}\right)$ and

$$
\left\{\begin{array}{c}
\|v\|_{L^{r_{1}\left([0, T], \dot{B}_{p_{1}, 2}^{\sigma}\right)}} \leq C\|\|_{0} \|_{\dot{H}^{\mu}} \\
\|w\|_{L^{r_{1}\left([0, T], \dot{B}_{p_{1}, 2}^{\sigma}\right)}} \leq C\|f\|_{L^{s_{1}\left([0, T], \dot{B}_{q_{1}, 2}^{\sigma}\right)}}
\end{array}\right.
$$


provided that

$$
\left\{\begin{array}{c}
\mu=\rho+1-2 / p_{1}=\sigma+1-2 / q_{1}, \\
1 / r_{1}+1 / p_{1}=1 / 2, \quad 2 \leq p_{1}<\infty, \quad 1 / s_{1}+1 / q_{1}=3 / 2, \quad 1<q_{1} \leq 2 .
\end{array}\right.
$$

In the case $\rho=\mu, p_{1}=2, r_{1}=\infty, v$ and $w$ belong $C^{0}\left([0, T], \dot{H}^{\mu}\right)$.

3) To prove (5.10), we use the linearity of (5.8) and split $u$ and the data into several pieces. First, we note that the low frequencies of $u$ are controlled by the $L^{2}$ norm. Thus the estimate (5.10) for $S_{0} u$ immediately follows from (5.9).

4) Consider the solution of (5.8) with $f=0$ and initial data $\left(1-S_{0}\right) u_{0}=0$. Then, thanks to the form (5.13) of the fundamental solution, the estimates (5.16) imply that

$$
\|u\|_{C^{0}\left([0, T] ; H^{\mu}\right)}+\|u\|_{L^{r}\left([0, T] ; B_{p, 2}^{0}\right)} \leq C\left\|u_{0}\right\|_{H^{\mu}} .
$$

We have replaced the homogeneous spaces by the inhomogeneous ones, using that the spectrum of $u$ is contained in $|\xi| \geq 1$. The same remark holds below when we use again (5.16). Moreover, since $L u=0$, one has

$$
\left\|\partial_{t} u\right\|_{C^{0}\left([0, T] ; H^{\mu-1}\right)} \leq C\|u\|_{C^{0}\left([0, T] ; H^{\mu}\right)},
$$

and (5.10) is satisfied.

5) Split $f$ into $f_{1}+f_{2}$ such that (5.6) (5.7) hold. Consider the solution of (5.8) with right hand side $\left(1-S_{0}\right) f_{1}$ and vanishing initial data. Then (5.16) implies that

$$
\|u\|_{C^{0}\left([0, T] ; H^{\mu}\right)}+\|u\|_{L^{r}\left([0, T] ; B_{p, 2}^{0}\right)} \leq C\left\|f_{1}\right\|_{L^{1}\left([0, T] ; H^{\mu}\right)}
$$

To estimate $\partial_{t} u$ one uses the equation and the inequality $\left\|f_{1}\right\|_{C^{0}\left([0, T] ; H^{\mu-1}\right)} \leq$ $\left\|f_{1}\right\|_{C^{0}\left([0, T] ; L^{2}\right)}$. This implies (5.10).

6) With $f_{2}$ satisfying (5.7), consider the solution $u$ of (5.8) with right hand side $\left(1-S_{0}\right) f_{2}$ and vanishing initial data. Using the fundamental solution (5.15), one gets that $u=u_{+}+u_{-}$, whose spatial Fourier transforms are given by

$$
\widehat{u}_{ \pm}(t, \xi)=\int_{0}^{t} e^{i(t-s)|\xi|} \widehat{f}_{ \pm}(s, \xi) d s, \quad f_{ \pm}:=\left(1-S_{0}\right) P_{ \pm} f_{2} .
$$

Integrating by parts shows that $u_{+}=v+w$ with

$$
\widehat{v}(t, \xi)=\int_{0}^{t} e^{i(t-s)|\xi|} \widehat{g}(s, \xi) d s, \quad \widehat{g}(s, \xi):=-\frac{i}{|\xi|} \partial_{t} \widehat{f}_{ \pm}(s, \xi)
$$

and

$$
w(t)=-G_{+}(t) h(0)+h(t), \quad \widehat{h}(t, \xi):=\frac{i}{|\xi|} \widehat{f}_{ \pm}(s, \xi)
$$


The assumption (5.7) implies that $\partial_{t} f_{2}$ is a sum of two terms $f_{2}^{\prime}+f_{2}^{\prime \prime}$ with the indicated regularity. Accordingly, one has $f_{+}=f_{+}^{\prime}+f_{+}^{\prime \prime}, g=g^{\prime}+g^{\prime \prime}$ and $v=v^{\prime}+v^{\prime \prime}$. The estimates (5.16) imply that

$$
\left\|v^{\prime}\right\|_{C^{0}\left([0, T] ; H^{\mu}\right) \cap L^{r}\left([0, T] ; B_{p, 2}^{0}\right)} \leq C\left\|g^{\prime}\right\|_{L^{1}\left([0, T] ; H^{\mu}\right)} \leq C^{\prime}\left\|f_{2}^{\prime}\right\|_{L^{1}\left([0, T] ; H^{\mu-1}\right)}
$$

and

$$
\left\|v^{\prime \prime}\right\|_{C^{0}\left([0, T] ; H^{\mu}\right) \cap L^{r}\left([0, T] ; B_{p, 2}^{0}\right)} \leq C\left\|g^{\prime \prime}\right\|_{L^{s}\left([0, T] ; B_{q, 2}^{1}\right)} \leq C^{\prime}\left\|f_{2}^{\prime \prime}\right\|_{L^{s}\left([0, T] ; B_{q, 2}^{0}\right)} .
$$

For the boundary term $w$, we have

$$
\begin{aligned}
\|w\|_{C^{0}\left([0, T] ; H^{\mu}\right) \cap L^{r}\left([0, T] ; B_{p, 2}^{0}\right)} & \leq 2\|h\|_{C^{0}\left([0, T] ; H^{\mu}\right) \cap L^{r}\left([0, T] ; B_{p, 2}^{0}\right)} \\
& \leq C\left\|f_{2}\right\|_{C^{0}\left([0, T] ; L^{2}\right) \cap L^{r}\left([0, T] ; B_{p, 2}^{-1}\right.} .
\end{aligned}
$$

Adding up the different estimates above and using the equation to estimate $\partial_{t} u_{+}$ yields

$$
\left\|u_{+}\right\|_{Y^{\mu}(T)} \leq C\|f\|_{Z^{\mu}(T)} .
$$

The estimate for $u_{-}$is similar and thus (5.10) is satisfied. This finishes the proof of Lemma 5.2.

Proof of Lemma 5.3. To simplify notations, we assume that $a$ and $u$ are scalar functions. For smooth functions, the product $a u$ can be split into two pieces (see $[\mathrm{Bo}])$

$$
a u=\widetilde{\Pi}(a, u)+\Pi(u, a):=\sum_{k=0}^{+\infty} S_{k+2} a \Delta_{k} u+\sum_{k=3}^{+\infty} S_{k-3} u \Delta_{k} a .
$$

Here the notations are slightly different from those used in (5.13). From now on, by definition, $\Delta_{0}=S_{0}$. We prove that $\widetilde{\Pi}(a, u)$ and $\Pi(u, a)$ extend as bilinear operators acting on functions $a$ which satisfy (5.3) and $u \in Y^{\mu}$, so that they satisfy (5.6) and (5.7) respectively.

1) For fixed $t$ and $\sigma \geq 0$, let us prove that

$$
\|\widetilde{\Pi}(a, u)(t)\|_{H^{\sigma}} \leq C\|a(t)\|_{L^{\infty}}\|u(t)\|_{H^{\sigma}} .
$$

For $\sigma>0,(5.18)$ follows from the estimate

$$
\left\|S_{k+2} a \Delta_{k} u\right\|_{L^{2}} \leq\left\|S_{k+2} a\right\|_{L^{\infty}}\left\|\Delta_{k} u\right\|_{L^{2}}
$$

and the fact that the spectrum of $S_{k+2} a \Delta_{k} u$ is contained in the ball $\left\{|\xi| \leq 2^{k+4}\right\}$. For $\sigma=0$ the proof is much more delicate. It is a classical result in harmonic analysis which can be found for instance in $[\mathrm{CM}]$. 
For all $\rho<\infty,(5.3)$ implies that $a \in C^{0}\left([0, T], L^{\rho}\right)$. This implies that for all $u \in C^{0}\left([0, T], H^{\mu}\right)$, the series $\sum_{k} S_{k+2} a \Delta_{k} u$ converges in $C^{0}\left([0, T], H^{\sigma}\right)$ for all $\sigma<\mu$ and the partial sums are bounded in $C^{0}\left([0, T], H^{\mu}\right)$. Therefore the sum belongs to $C^{0}\left([0, T], L^{2}\right) \cap L^{\infty}\left([0, T], H^{\mu}\right)$. This shows that $\widetilde{\Pi}(a, u)$ extends to the $a$ 's which satisfy $(5.3)$ and $u \in C^{0}\left([0, T], H^{\mu}\right)$. Moreover, $\widetilde{\Pi}(a, u) \in C^{0}\left([0, T], L^{2}\right) \cap$ $L^{1}\left([0, T], H^{\mu}\right)$ and (5.18) implies that

$$
\|\widetilde{\Pi}(a, u)\|_{C^{0}\left([0, T], L^{2}\right) \cap L^{1}\left([0, T], H^{\mu}\right)} \leq C\|a\|_{L^{\infty}}\left(T\|u\|_{C^{0}\left([0, T] ; H^{\mu}\right)}+\|u\|_{C^{0}\left([0, T] ; L^{2}\right)}\right) .
$$

2) For fixed $t$ and $\sigma \leq 0$, let us show that

$$
\|\Pi(u, a)(t)\|_{H^{\sigma}} \leq C\|a(t)\|_{L^{\infty}}\|u(t)\|_{H^{\sigma}} .
$$

The proof for $\sigma<0$ is easy, using that $\left\|S_{k-3} u \Delta_{k} a\right\|_{L^{2}} \leq \sum_{j<k-3}\left\|\Delta_{j} u\right\|_{L^{2}}$ $\left\|\Delta_{k} a\right\|_{L^{\infty}}$ and the fact that the spectrum of $S_{k+2} a \Delta_{k} u$ is contained in the annulus $\left\{2^{k-2} \leq|\xi| \leq 2^{k+2}\right\}$. The result for $\sigma=0$ is proved in [CM]. It is equivalent to (5.18) with $\sigma=0$ since the product au has an obvious estimate in $L^{2}$. In addition, since $a \in C^{0}\left([0, T], L^{\rho}\right)$ for all $\rho<\infty$ and $u \in C^{0}\left([0, T], H^{\mu}\right) \subset C^{0}\left([0, T], L^{\rho^{\prime}}\right)$ for some $\rho^{\prime}>2$, it follows that $a u \in C^{0}\left([0, T], L^{2}\right)$. Thus $\Pi(u, a)=a u-\widetilde{\Pi}(a, u) \mathrm{ex}-$ tends to $a$ satisfying $(5.3)$ and $u \in C^{0}\left([0, T], H^{\mu}\right)$ so that $\Pi(u, a) \in C^{0}\left([0, T], L^{2}\right)$. Moreover, (5.20) implies that

$$
\|\Pi(u, a)\|_{C^{0}\left([0, T], L^{2}\right)} \leq C\|a\|_{L^{\infty}}\|u\|_{C^{0}\left([0, T], L^{2}\right)} .
$$

For $p \geq 2$, one has $\left\|\Delta_{j} u\right\|_{L^{p}} \leq C 2^{3 j(1 / 2-1 / p)}\left\|\Delta_{j} u\right\|_{L^{p}}$. Thus $1-2 / p=\mu$ yields

$$
\left\|S_{k-3} u \Delta_{k} a\right\|_{L^{p}} \leq \sum_{j \leq k-3}\left\|\Delta_{j} u\right\|_{L^{p}}\left\|\Delta_{k} a\right\|_{L^{\infty}} \leq C\|a\|_{L^{\infty}} \sum_{j \leq k-3} 2^{3 j \mu / 2}\left\|\Delta_{j} u\right\|_{L^{2}} .
$$

Since the spectrum of $S_{k+2} a \Delta_{k} u$ is contained in $\left\{2^{k-2} \leq|\xi| \leq 2^{k+2}\right\}$, it follows that

$$
\|\Pi(u, a)(t)\|_{B_{p, 2}^{-\mu / 2}} \leq C\|a(t)\|_{L^{\infty}}\|u(t)\|_{H^{\mu}} .
$$

Therefore $1 / r=\mu / 2$ yields

$$
\|\Pi(u, a)\|_{L^{r}\left([0, T] ; B_{p, 2}^{-1}\right)} \leq C T^{\mu / 2}\|a\|_{L^{\infty}}\|u\|_{C^{0}\left([0, T] ; H^{\mu}\right)} .
$$

This estimate also holds for the extended definition of $\Pi(u, a)$, since the space in the left hand side is a dual and (5.22) provides uniform estimates for approximations of $a$ and $u$.

3) So far, we have proved that $\Pi(u, a) \in C^{0}\left([0, T] ; L^{2}\right) \cap L^{r}\left([0, T] ; B_{p, 2}^{-1}\right)$. We now study $\partial_{t} \Pi(u, a)$. For smooth $a$ and $u$, one has

$$
\partial_{t} \Pi(a u)=\Pi\left(\partial_{t} u, a\right)+\Pi\left(u, \partial_{t} a\right) .
$$


The estimate (5.20) shows that

$$
\left\|\Pi\left(\partial_{t} u, a\right)\right\|_{L^{\infty}\left([0, T] ; H^{\mu-1}\right)} \leq C\|a\|_{L^{\infty}}\left\|\partial_{t} u\right\|_{C^{0}\left([0, T] ; H^{\mu-1}\right)} .
$$

For the second term, use the relation $1 / p+1 / 2=1 / q$ to find

$$
\left\|S_{k-3} u \Delta_{k} \partial_{t} a\right\|_{L^{q}} \leq\left\|S_{k-3} u\right\|_{L^{p}}\left\|\Delta_{k} \partial_{t} a\right\|_{L^{2}} .
$$

Since $p \geq 2, B_{p, 2}^{0} \subset L^{p}$ (see e.g. [Tr]). Thus $\left\|S_{k} u(t)\right\|_{L^{p}} \leq C\|u(t)\|_{L^{p}} \leq$ $C^{\prime}\|u(t)\|_{B_{p, 2}^{0}}$. Since the spectrum of $S_{k-3} u \Delta_{k} a$ is contained in $\left\{2^{k-2} \leq|\xi| \leq\right.$ $\left.2^{k+2}\right\}$, this shows that

$$
\left\|\Pi\left(u, \partial_{t} a\right)(t)\right\|_{B_{q, 2}^{0}} \leq C\left\|\partial_{t} a(t)\right\|_{L^{2}}\|u(t)\|_{B_{p, 2}^{0}}
$$

and, with $1 / s-1 / r=1 / 2$, that

$$
\left\|\Pi\left(u, \partial_{t} a\right)\right\|_{L^{s}\left([0, T] ; B_{q, 2}^{0}\right)} \leq C T^{1 / 2}\left\|\partial_{t} a\right\|_{L^{\infty}\left([0, T], L^{2}\right)}\|u\|_{L^{r}\left([0, T] ; B_{p, 2}^{0}\right)} .
$$

The spaces in the left hand sides of (5.24) and (5.25) are dual spaces. Thus the bilinear operators $\Pi\left(\partial_{t} u, a\right)$ and $\Pi\left(u, \partial_{t} a\right)$ extend to the spaces which occur in the right hand sides. This shows that for $a$ satisfying (5.3) and $u \in Y^{\mu}(T)$, one gets that $\partial_{t} \Pi(u, a) \in L^{1}\left([0, T], H^{\mu-1}\right)+L^{s}\left([0, T], B_{q, 2}^{0}\right)$ and

$$
\begin{gathered}
\left\|\partial_{t} \Pi(u, a)\right\|_{L^{1}\left([0, T], H^{\mu-1}\right)+L^{s}\left([0, T] ; B_{q, 2}^{0}\right)} \leq C T\|a\|_{L^{\infty}}\left\|\partial_{t} u\right\|_{C^{0}\left([0, T] ; H^{\mu-1}\right)} \\
+C T^{1 / 2}\left\|\partial_{t} a\right\|_{L^{\infty}\left([0, T], L^{2}\right)}\|u\|_{L^{r}\left([0, T] ; B_{p, 2}^{0}\right)} .
\end{gathered}
$$

Together with (5.19), (5.21) and (5.22), this finishes the proof of Lemma 5.3.

Proof of Theorem 2.7, when $\mu=1$. Consider a solution $U$ of (1.2) (1.3) with Cauchy data $E_{0} \in H^{1}$ and $H_{0 \perp} \in H^{1}$. Theorem 2.7 with $\mu=1 / 2$ implies that $H_{\perp} \in C^{0}\left(\left[0,+\infty\left[; H^{1 / 2}\right)\right.\right.$. The Sobolev embedding $H^{1 / 2} \subset L^{3}$ implies that

$$
\partial_{t} M=F(M, H)=F\left(M, H_{\perp}\right)-F\left(M, M_{\|}\right) \in L_{l o c}^{\infty}\left(\left[0,+\infty\left[; L^{3}\right) .\right.\right.
$$

Therefore, the coefficient $a$ and the source term $g$ in (5.1) or (5.2) satisfy, in addition to (5.3) (5.4),

$$
\partial_{t} a \in L^{\infty}\left([0, T] ; L^{3}\right), \quad \partial_{t} g \in L^{\infty}\left([0, T] ; L^{2}\right)
$$

for all $T>0$. Again, we consider $u=\left(E_{\perp}, H_{\perp}\right)$ as the unique solution to the linear problem (5.2) and prove that if the initial data belong to $H^{1}$, then there is a solution in $C^{0}\left([0, T], H^{1}\right)$ for all $T>0$. 
1) For $f \in L^{1}\left([0, T], L^{2}\right)$, the solution to (5.8) satisfies

$$
\|u(t)\|_{L^{2}} \leq\|u(0)\|_{L^{2}}+2 \int_{0}^{t}\|f(s)\|_{L^{2}} d s .
$$

If $f \in C^{0}\left([0, T], L^{2}\right)$ and $\partial_{t} f \in L^{1}\left([0, T], L^{2}\right)$, then, using the fundamental solution (5.13) and integrating by parts as in the proof of Lemma 5.2, one obtains

$$
\left\|\partial_{x} u(t)\right\|_{L^{2}} \leq\left\|\partial_{x} u(0)\right\|_{L^{2}}+C\left(\int_{0}^{t}\left\|\partial_{t} f(s)\right\|_{L^{2}} d s+\|f(0)\|_{L^{2}}+\|f(t)\|_{L^{2}}\right) .
$$

Moreover

$$
\left\|\partial_{t} u(t)\right\|_{L^{2}} \leq C\left\|\partial_{x} u(t)\right\|_{L^{2}}+\|u(t)\|_{L^{2}} .
$$

2) One has

$$
\|a(t) u(t)\|_{L^{2}} \leq\|a\|_{L^{\infty}}\|u(t)\|_{L^{2}} .
$$

Using the Sobolev inequality $\|u\|_{L^{6}} \leq C\left\|\partial_{x} u\right\|_{L^{2}}$, one gets

$$
\left\|\partial_{t}(a u)(t)\right\|_{L^{2}} \leq\|a\|_{L^{\infty}}\left\|\partial_{t} u(t)\right\|_{L^{2}}+\left\|\partial_{t} a\right\|_{L^{2}}\left\|\partial_{x} u(t)\right\|_{L^{2}} .
$$

3) Let $K$ denote the operator $v \mapsto u$, where $u$ is the solution of (5.8) with source term $f=a v$ and vanishing initial data. The estimates above show that $K$ maps $C^{0}\left([0, T] ; H^{1}\right) \cap C^{1}\left([0, T], L^{2}\right)$ into itself and

$$
\begin{gathered}
\|K v(t)\|_{L^{2}} \leq C \int_{0}^{t}\|v(s)\|_{L^{2}} d s \\
\left\|\partial_{t, x} K v(t)\right\|_{L^{2}} \leq C\left(\int_{0}^{t}\left\|\partial_{t, x} v(s)\right\|_{L^{2}} d s+\|v(t)\|_{L^{2}}+\|v(0)\|_{L^{2}}\right) .
\end{gathered}
$$

where $C$ only depends on $a$.

4) The first two steps imply that the solution to (5.8), with source term $g$ and initial data $u_{0}$, belongs to $C^{0}\left([0, T] ; H^{1}\right) \cap C^{1}\left([0, T], L^{2}\right)$. The third step implies that Picard's iterates converge in $C^{0}\left([0, T] ; H^{1}\right) \cap C^{1}\left([0, T], L^{2}\right)$, proving that the unique solution to $(5.2)$ also belongs to $C^{0}\left([0, T] ; H^{1}\right) \cap C^{1}\left([0, T], L^{2}\right)$. The proof of Theorem 2.7 is now complete.

\section{Uniqueness and $L^{2}$-stability of the $H_{\text {curl }}$ solution}

In this section we prove Theorem 2.8. It follows from a stronger result on the stability of finite energy solutions $\underline{U}$ such that $\operatorname{curl} \underline{E}$ and $\operatorname{curl} \underline{H}$ belong to $C^{0}([0,+\infty[$; $\left.L^{2}\left(\mathbf{R}^{3}\right)\right)$. Before stating the result, we make a few remarks. satisfies

Consider two finite energy solutions $\underline{U}$ and $U$ on $\Omega_{T}$. Then $\delta U=U-\underline{U}$

$$
L \delta U:=\left(\begin{array}{c}
0 \\
-\delta F \\
\delta F
\end{array}\right)
$$


where $L$ denotes the first-order system in $(1.2)$ and $\delta F=F(M, H)-F(\underline{M}, \underline{H})=$ $F(M, H-\underline{H})+F(M, \underline{H})-F(\underline{M}, \underline{H})$. Suppose that $\|M(0)\|_{L^{\infty}} \leq R$ and $\|M(0)\|_{L^{\infty}} \leq R$. Then, $\|M\|_{L^{\infty}} \leq R$ and $\|M\|_{L^{\infty}} \leq R$. Suppose in addition that

$$
\|\underline{H}\|_{L^{\infty}\left(\Omega_{T}\right)}<\infty .
$$

Then $|\delta F| \leq C|\delta U|$, where $C$ depends on $R$ and $\|\underline{H}\|_{L^{\infty}\left(\Omega_{T}\right)}$. The standard energy estimate for (6.1) implies that

$$
\|\delta U(t)\|_{L^{2}} \leq e^{C t}\|\delta U(0)\|_{L^{2}}
$$

proving that $\underline{U}$ is the unique finite energy solution with initial data $\underline{U}(0)$. The uniqueness of bounded solutions of semilinear equations is well known. Here, we have a-priori bounds of the $L^{\infty}$ norms of $M$ and $\underline{M}$. The interesting point is that (6.2) involves only $\underline{H}$.

The main goal of this section is to weaken condition (6.2). The price is the lost of the Lipschitz dependence of $U(t)$ on $U(0)$.

Theorem 6.1 Let $\underline{U}$ be a finite energy solution on $\Omega_{T}$ such that $\|M(0)\|_{L^{\infty}}$ $\leq R$, curl $\underline{E}$ and $\operatorname{curl} \underline{H}$ belong to $C^{0}\left([0, T] ; L^{2}\left(\mathbf{R}^{3}\right)\right)$. Then there exist constants $C>0, c>0$ and $\rho>0$, such that for all finite energy solution $U$ on $\Omega_{T}$ which satisfies $\|M(0)\|_{L^{\infty}} \leq R$ and $\|U(0)-\underline{U}(0)\|_{L^{2}} \leq c$, one has for all $t \in[0, T]$

$$
\|U(t)-\underline{U}(t)\|_{L^{2}} \leq C\|U(0)-\underline{U}(0)\|_{L^{2}}^{\gamma(t)}
$$

with $\gamma(t):=e^{-\rho t}$

The main ingredient is a substitute to the $L^{\infty}$ estimate (6.2).

Lemma 6.2 There is a constant $C$ such that for all $\lambda \geq e$, there is $\underline{H}^{\lambda} \in L^{\infty}\left(\Omega_{T}\right)$ and functions $\alpha_{\lambda} \in L^{2}([0, T]), \beta_{\lambda} \in L^{\infty}([0, T])$ such that for all $t \in[0, T]$

$$
\begin{array}{r}
\left\|\underline{H}^{\lambda}(t)\right\|_{L^{\infty}} \leq \alpha_{\lambda}(t)+\beta_{\lambda}(t), \quad\left\|\left(\underline{H}-\underline{H}^{\lambda}\right)(t)\right\|_{L^{2}} \leq C / \lambda, \\
\left\|\alpha_{\lambda}\right\|_{L^{2}([0, T])} \leq C \sqrt{\ln \lambda}, \quad\left\|\alpha_{\lambda}\right\|_{L^{\infty}([0, T])} \leq C \ln \lambda .
\end{array}
$$

Proof. Using (2.13) we write $\underline{H}=\underline{H}_{\perp}-\underline{M}_{\|}$and study each term separately.

1) The operator $P_{\|}$maps $L^{p}$ in $L^{p}$ for all finite $p$, with norm less or equal to $C_{0} p$, with $C_{0}$ independent of $p$ (see [St] for instance). Therefore, for all $p \in[2,+\infty[$,

$$
\left\|\underline{M}_{\|}(t)\right\|_{p} \leq C_{0} p\|\underline{M}(t)\|_{L^{2} \cap L^{\infty}} \leq C_{0} p\|\underline{M}(0)\|_{L^{2} \cap L^{\infty}} .
$$

Define $\underline{M}_{\|}^{\lambda}(t, x)=\underline{M}_{\|}(t, x)$ when $\left|\underline{M}_{\|}(t, x)\right| \leq C \ln \lambda$ and $\underline{M}_{\|}^{\lambda}(t, x)=0$ otherwise. Then

$$
\left\|\left(\underline{M}_{\|}-\underline{M}_{\|}^{\lambda}\right)(t)\right\|_{L^{2}}^{2} \leq \frac{1}{(C \ln \lambda)^{p-2}}\left\|\underline{M}_{\|}(t)\right\|_{L^{p}}^{p} \leq \frac{\left(C_{1} p\right)^{p}}{(C \ln \lambda)^{p-2}}
$$

with $C_{1}=C_{0}\|\underline{M}(0)\|_{L^{2} \cap L^{\infty}}$. Choose $C=2 e C_{1}$ and $p=2 \ln \lambda \geq 2$. Then the right hand side is less than

$$
(C \ln \lambda)^{2} e^{-p}=(C \ln \lambda)^{2} \lambda^{-2} \leq C^{\prime} \lambda^{-1} .
$$


2) Applying $P_{\perp} \partial_{t}$ to the second equation in (1.2) yields

$$
\square \underline{H}_{\perp}=-P_{\perp} \partial_{t} F(\underline{M}, \underline{H}) .
$$

Since $F$ is Lipschitzean and $\left(\partial_{t} \underline{M}, \partial_{t} \underline{H}\right) \in L^{2}$, one can differentiate $F(\underline{M}, \underline{H})$ with respect to $t$. Using (2.4), one obtains that

$$
\left|\partial_{t} F(\underline{M}, \underline{H})\right| \leq C\left(\left|\partial_{t} \underline{H}\right|+|\underline{H}|\left|\partial_{t} \underline{M}\right|\right) \leq C\left(\left|\partial_{t} \underline{H}\right|+|\underline{H}|^{2}\right) .
$$

We know that $\partial_{t} \underline{H}=-\operatorname{curl} \underline{E}-F(\underline{M}, \underline{H}) \in L^{1}\left([0, T] ; L^{2}\right)$. Moreover, $\underline{M}_{\|}(t)$ is bounded in $L^{4}$ and $\underline{H}_{\perp}(t)$ is bounded in $H^{1}$ thus in $L^{4}$. Therefore $\underline{H}(t)=$ $\underline{H}_{\perp}(t)-\underline{M}_{\|}(t)$ is bounded in $L^{4}$ and the right hands side of (6.7) belongs to $L^{1}\left([0, T] ; L^{2}\right)$. In addition, the initial values of $\underline{H}_{\perp}$ satisfy

$$
\underline{H}_{\perp}(0) \in H^{1}, \quad \partial_{t} \underline{H}_{\perp}(0)=-\operatorname{curl} \underline{E}(0)-F(\underline{M}(0), \underline{H}(0)) \in L^{2} .
$$

One would like to use the Stichartz estimates to control the $L^{2}\left(L^{\infty}\right)$ norm of $\underline{H}_{\perp}$ by the $L^{1}\left(L^{2}\right)$ norm of $\square \underline{H}_{\perp}$. This corresponds to the forbidden limit case $p_{1}=\infty$ in (5.16) for which the inequality is known to be false (see $[\mathrm{L}],[\mathrm{KM}])$. Nevertheless we persist in following this idea. The Strichartz inequality in the limit case $p_{1}=\infty$ holds for functions whose Fourier transform is supported in a ball and it is possible to give a sharp estimate of the constant involved in term of the radius of the ball. Precisely, recalling the definition of $S^{\lambda}$ in (2.9), we have

Proposition 6.3 There exists a constant $c$ such that for all $\lambda>0$, all $T>0$ and all $u \in C^{0}\left(\left[0, \infty\left[; H^{2}\left(\mathbf{R}^{3}\right)\right)\right.\right.$,

$$
\left\|S^{\lambda} u\right\|_{L^{2}\left([0, T] ; L^{\infty}\left(\mathbf{R}^{3}\right)\right)} \leq c \sqrt{\log (1+\lambda T)}\left(\left\|\partial_{t, x} u(0)\right\|_{L^{2}\left(\mathbf{R}^{3}\right)}+\|\square u\|_{L^{1}\left([0, T] ; L^{2}\left(\mathbf{R}^{3}\right)\right)}\right) .
$$

The proof is delayed until section 8. A similar idea would be to estimate the constant $C$ in (5.16) as $p_{1} \rightarrow \infty$. It would lead to similar results.

This proposition applies to $\underline{H}_{\perp}$. Therefore the function $\alpha_{\lambda}(t):=$ $\left\|S^{\lambda} \underline{H}_{\perp}(t)\right\|_{L^{\infty}}$ belongs to $L^{2}([0, T])$ and

$$
\left\|\alpha_{\lambda}\right\|_{L^{2}([0, T])} \leq C \sqrt{\ln (1+\lambda T)} \leq C^{\prime} \sqrt{\ln \lambda}
$$

for $\ln \lambda \geq 1$. Next, we note that $\underline{H}_{\perp}-S^{\lambda} \underline{H}_{\perp}$ satisfies

$$
\left\|\left(I-S^{\lambda}\right) \underline{H}_{\perp}(t)\right\|_{L^{2}} \leq \frac{1}{2 \lambda}\left\|\underline{H}_{\perp}(t)\right\|_{H^{1}} \leq C / \lambda .
$$

3) The Lemma 6.2 follows, with $\underline{H}^{\lambda}=S^{\lambda} \underline{H}_{\perp}-\underline{M}_{\|}^{\lambda}, \alpha_{\lambda}(t):=\left\|S^{\lambda} \underline{H}_{\perp}(t)\right\|_{L^{\infty}}$ and $\beta_{\lambda}(t):=\left\|\underline{M}_{\|}^{\lambda}(t)\right\|_{L^{\infty}}$. 
Proof of Theorem 6.1. Using the inequalities (2.4) for the function $F$ and the $L^{\infty}$ bounds (2.6) for $M$ and $\underline{M}$, one obtains that the right hand side $\delta F=F(M, \delta H)+$ $F(M, \underline{H})-F(\underline{M}, \underline{H})$ in $(6.1)$ satisfies

$$
|\delta F| \leq C(R)(|\delta H|+|\underline{H}||\delta M|) .
$$

For all $\lambda \geq e$, choose $\underline{H}^{\lambda}$ as indicated in Lemma 6.2. Then, (6.9) implies that

$$
\|\delta F(t)\|_{L^{2}} \leq C\left(\|\delta H(t)\|_{L^{2}}+\left(\alpha_{\lambda}(t)+\beta_{\lambda}(t)\right)\|\delta M(t)\|_{L^{2}}+\frac{1}{\lambda}\|\delta M(t)\|_{\infty}\right) .
$$

With the obvious estimate $|\delta M| \leq|M|+|\underline{M}| \leq 2 R$, this implies that

$$
\|\delta F(t)\|_{L^{2}} \leq C\left(\left(1+\alpha_{\lambda}(t)+\beta_{\lambda}(t)\right)\|\delta U(t)\|_{L^{2}}+\frac{1}{\lambda}\right) .
$$

for some $C$ that depends only on $\|\underline{M}(0)\|_{L^{2} \cap L^{\infty}}, R$ and $\|\operatorname{curl} \underline{H}\|_{C^{0}\left([0, T] ; H^{1}\left(\mathbf{R}^{3}\right)\right)}$. Introduce

$$
\delta(t)=\|\delta U(t)\|_{L^{2}} .
$$

The energy estimate for (6.1) together with (6.10) yields

$$
\delta(t) \leq \delta(0)+2 C \int_{0}^{t}\left(\left(1+\alpha_{\lambda}(s)+\beta_{\lambda}(s)\right) \delta(s)+\frac{1}{\lambda}\right) d s .
$$

Gronwall's Lemma implies that for all $\lambda \geq e$ and all $t \in[0, T]$

$$
\delta(t) \leq\left(\delta(0)+\frac{C t}{\lambda}\right) e^{A_{\lambda}(t)}
$$

where

$$
A_{\lambda}(t):=2 C \int_{0}^{t}\left(1+\alpha_{\lambda}(s)+\beta_{\lambda}(s)\right) d s .
$$

Lemma 6.2 implies that for all $\lambda \geq e$ and $0 \leq t \leq T$,

$$
A_{\lambda}(t) \leq C(t \ln \lambda+\sqrt{t \ln \lambda}) \leq C_{1}(1+t \ln \lambda)
$$

Suppose that $\lambda>1 / \delta(0)$. Then (6.11) and (6.12) imply

$$
\delta(t) \leq \delta(0)(1+C t) e^{C_{1}(1+t \ln \lambda)} .
$$

Suppose that $\delta(0)<1 / e$. For $t \leq T_{1}:=1 / 2 C_{1}$, one can let $\lambda \rightarrow 1 / \delta(0)$ to find

$$
\delta(t) \leq e^{C_{1}}\left(1+C T_{1}\right) \delta(0)^{1-t C_{1}} .
$$

Introduce $\rho=C_{1} \ln 4$. Then $1-t C_{1} \geq \gamma(t):=e^{-\rho t}$ for $t \leq 1 / 2 C_{1}$. Summing up, we have shown that there are constant $T_{1}>0, C_{2}$ and $\rho$ such that, if $\delta(0) \leq 1 / e$, then for $t \leq T_{1}$ :

$$
\delta(t) \leq C_{2} \delta(0)^{\gamma(t)}
$$

If $C_{2} \delta(0)^{\gamma\left(T_{1}\right)} \leq 1 / e$, one can apply (6.14) to the Cauchy problem with initial time $T_{1}$ and prove that (6.14) with another constant $C_{2}$ holds on $\left[T_{1}, 2 T_{1}\right]$. By induction this implies that for $\delta(0)$ small enough, the estimate (6.4) follows. 


\section{Global smooth solutions}

In this section, we prove Theorem 2.9. We therefore assume that $F$ satisfies Assumption 2.1 and in particular is infinitely smooth. Classical results for the semilinear Cauchy problem in $\mathbf{R}^{1+3}$ with initial data $U_{0}$ in $H^{s}, s \geq 2$, say that there exists a unique maximal solution $U \in C^{0}\left(\left[0, T\left[; H^{s}\right)\right.\right.$ of $(1.2)$ satisfying $U(0)=U_{0}$. Moreover, if $T<\infty$, then $\|U(t)\|_{L^{\infty}} \rightarrow \infty$, and hence $\|U(t)\|_{H^{2}} \rightarrow \infty$ as $t \rightarrow T$. Therefore, Theorem 2.9 is a consequence of the following a priori estimate.

Proposition 7.1 Let $F$ satisfy Assumption 2.1. For all $T>0$, any finite energy solution $U$ in $\Omega_{T}$ belonging to $C^{0}\left(\left[0, T\left[; H^{2}\left(\mathbf{R}^{3}\right)\right)\right.\right.$, satisfies

$$
\sup _{0 \leq t<T}\|U(t)\|_{H^{2}\left(\mathbf{R}^{3}\right)} \leq C
$$

where $C$ depends only on $T$ and $\|U(0)\|_{H^{2}\left(\mathbf{R}^{3}\right)}$.

Let $\partial U$ denote any $x$-derivative of $U$. Differentiating twice the equations for $U$ leads to a system for $U, \partial U$ and $\partial^{2} U$ which reads

$$
L U=f_{0}, \quad L \partial U=f_{1}, \quad L \partial^{2} U=f_{2} .
$$

The nonlinear part of $f_{i}, i=1,2$ involve the first and second order derivatives of $F$ with respect to $m$. Assumption 2.1, the chain rule and the uniform bound (2.6) for $M$ immediately yield the following pointwise estimates which hold almost everywhere on $\Omega_{T}$ :

$$
\left\{\begin{array}{c}
\left|f_{0}\right| \leq C|H|, \quad\left|f_{1}\right| \leq C(|\partial H|+|H||\partial M|) \\
\left|f_{2}\right| \leq C\left(\left|\partial^{2} H\right|+|\partial H||\partial M|+|H|\left|\partial^{2} M\right|+|H||\partial M|^{2}\right),
\end{array}\right.
$$

where $C$ is some constant that depends on $\|M(0)\|_{\infty}=R$. Introduce

$$
n_{2}(t)=\sup _{0 \leq s \leq t}\|U(s)\|_{H^{2}}
$$

In order to apply the energy estimate for the large system (7.1) we need to bound from above the $L^{2}$-norms of the $f_{i}(t)$ by some functions of $n_{2}(t)$. The linear terms in (7.2) are trivially bounded by $n_{2}(t)$. Thus it remains to control the quadratic and cubic terms. The key is to obtain bounds for the $L^{\infty}$ norm of $H$. For $H_{\|}=-M_{\|}$, we use a Judovic-type inequality.

Lemma 7.2 There exists $C$ depending only on $\|M(0)\|_{L^{2} \cap L^{\infty}}$ such that for all $t \in[0, T[$

$$
\left\|M_{\|}(t)\right\|_{\infty} \leq C\left(1+\ln ^{+}\left(\|M(t)\|_{H^{2}}\right)\right) .
$$

where $\ln ^{+}$denotes the positive part of $\ln$. 
Proof. Introduce a Littlewood-Paley decomposition $1=\varphi(\xi)+\sum_{k=1}^{\infty} \psi_{1}\left(2^{-k} \xi\right)$ as in (5.13) and use the notations $S_{k}:=\varphi\left(D_{x}\right), \Delta_{k}:=\psi_{k}\left(D_{x}\right)$. We first prove that for all $k \geq 1$,

$$
\left\|S_{k} M_{\|}\right\|_{L^{\infty}} \leq 2^{\frac{3}{2}}\|M\|_{L^{2}}+c k\|M\|_{L^{\infty}}
$$

where $c$ only depends on $\varphi$. One has

$$
\left\|S_{0} M_{\|}\right\|_{L^{\infty}} \leq 2^{\frac{3}{2}}\left\|S_{0} M_{\|}\right\|_{L^{2}} \leq 2^{\frac{3}{2}}\|M\|_{L^{2}}
$$

since $P_{\|}$is an orthogonal projector on $L^{2}$ and $\varphi$ is supported in $|\xi| \leq 2$. Denote by $p_{\|}(\xi)=\frac{1}{|\xi|^{2}}(\xi, \cdot) \xi$ the symbol of $P_{\|}$. Since $p_{\|} \psi_{1} \in C_{0}^{\infty}$, its Fourier transform $\chi(x)$ belongs to the Schwartz class $\mathcal{S}$. Since $p_{\|}$is homogeneous of degree 0 , it follows that, for all $k \geq 1, P_{\|} \circ \psi_{1}\left(2^{-k} D_{x}\right)$ is the convolution operator with $2^{3 k} \chi\left(2^{k} x\right)$, whose $L^{1}$-norm is independent of $k$. Thus, for all $k \geq 1$,

$$
\left\|\Delta_{k} M_{\|}\right\|_{L^{\infty}} \leq\|\chi\|_{L^{1}}\|M\|_{L^{\infty}}
$$

Since $S_{k}=S_{0}+\sum_{j \leq k} \Delta_{j}$, (7.6) and (7.7) imply (7.5). To end the proof of the lemma, note that in $\mathbf{R}^{3}$

$$
\left\|\left(I-S_{k}\right)(u)\right\|_{L^{\infty}} \leq c 2^{-k / 2}\left\|\partial^{2} u\right\|_{L^{2}}
$$

Since $P_{\|}$is orthogonal and commute with $\partial^{2}$, this implies

$$
\left\|M_{\|}-S_{k} M_{\|}\right\|_{L^{\infty}} \leq c 2^{-k / 2}\|M\|_{H^{2}}
$$

Let $\lambda:=\|M(t)\|_{H^{2}} /\|M(t)\|_{L^{\infty}}$, noticing that $\lambda \geq \sqrt{8 \pi}$. Choose $k$ such that $2^{k / 2} \leq$ $\lambda \leq 2^{(k+1) / 2}$. Then (7.5), (7.9) and the inequality $\|M(t)\|_{L^{\infty} \cap L^{2}} \leq\|M(0)\|_{L^{\infty} \cap L^{2}}$ imply (7.4).

Lemma 7.3 There are a universal constant $c$ and a constant $C_{1}$ which depends only on $\|M(0)\|_{L^{\infty} \cap L^{2}},\|\operatorname{curl}(E, H)(0)\|_{L^{2}}$ and $T$, such that for all $\lambda \geq e$, there is a function $\alpha_{\lambda} \in L^{2}([0, T])$ such that $\left\|\alpha_{\lambda}\right\|_{L^{2}([0, T])} \leq C_{1} \sqrt{\ln \lambda}$ and for all $t \in[0, T[$,

$$
\left\|H_{\perp}(t)\right\|_{L^{\infty}} \leq \alpha_{\lambda}(t)+\frac{c}{\sqrt{\lambda}}\|H(t)\|_{H^{2}} .
$$

Proof. By Theorems 2.7 and 2.8, we know that $U$ extends as a finite energy solution on $\Omega_{\infty}$, with $\left(E, H_{\perp}\right) \in C^{0}\left(\left[0, \infty\left[; H^{1}\right)\right.\right.$. Thus the norm of $\left(E, H_{\perp}\right)$ in $C^{0}\left([0, T] ; H^{1}\right)$ is bounded by a constant $C_{1}$ which depends only on $\|M(0)\|_{L^{\infty} \cap L^{2}}$, $\|\operatorname{curl}(E, H)(0)\|_{L^{2}}$ and $T$. As in the proof of Lemma 6.2, this implies that $\square H_{\perp} \in$ $L^{1}\left([0, T] ; L^{2}\right), H_{\perp}(0) \in H^{1}$ and $\partial_{t} H_{\perp}(0) \in L^{2}$, with norms less than a similar constant $C_{1}$. Therefore Proposition 6.3 implies that the function $t \mapsto\left\|S^{\lambda}\left(H_{\perp}(t)\right)\right\|_{L^{\infty}}$ belongs to $L^{2}([0, T])$ with norm less than $C_{1} \sqrt{\ln \lambda}$. To prove (7.10) write

$$
\left\|H_{\perp}(t)\right\|_{L^{\infty}} \leq\left\|S^{\lambda}\left(H_{\perp}(t)\right)\right\|_{L^{\infty}}+\left\|\left(I-S^{\lambda}\right)\left(H_{\perp}(s)\right)\right\|_{L^{\infty}}
$$

and recall (7.8), which implies that

$$
\left\|\left(I-S^{\lambda}\right)\left(H_{\perp}(t)\right)\right\|_{L^{\infty}} \leq c\left\|H_{\perp}(t)\right\|_{H^{2}} \leq c\|H(t)\|_{H^{2}} .
$$


Proof of Proposition 7.1. Let $U \in C^{0}\left(\left[0, T\left[; H^{2}\left(\mathbf{R}^{3}\right)\right)\right.\right.$ be a finite energy solution in $\Omega_{T}$. We estimate the $L^{2}$ norms of the $f_{i}(t)$, using (7.2). Recall the CagliardoNirenberg inequality

$$
\left\||\partial u|^{2}\right\|_{L^{2}} \leq 4\|u\|_{L^{\infty}}\left\|\partial^{2} u\right\|_{L^{2}}
$$

It implies that

$$
\||\partial H(t)||\partial M(t)|\|_{L^{2}} \leq 4\|H(t)\|_{L^{\infty}}^{1 / 2}\|M(t)\|_{L^{\infty}}^{1 / 2}\|U(t)\|_{H^{2}}
$$

and

$$
\left\||H(t)||\partial M(t)|^{2}\right\|_{L^{2}} \leq 4\|H(t)\|_{L^{\infty}}\|M(t)\|_{L^{\infty}}\|M(t)\|_{H^{2}} .
$$

Therefore, (7.2) implies that

$$
\left\|f_{i}(t)\right\|_{L^{2}} \leq C\left(1+\|H(t)\|_{L^{\infty}}\right)\|U(t)\|_{H^{2}} .
$$

where $C$ only depends on $\left\|M_{0}\right\|_{L^{2} \cap L^{\infty}}$. Next write $H=H_{\perp}-M_{\|}$and use Lemmas 7.2 and 7.3 to find

$$
\left\|f_{i}(t)\right\|_{L^{2}} \leq C\left(1+\alpha_{\lambda}(t)+\frac{\|U(t)\|_{H^{2}}}{\sqrt{\lambda}}+\ln ^{+}\left(\|U(t)\|_{H^{2}}\right)\right)\|U(t)\|_{H^{2}} .
$$

Therefore, the energy estimates for the large system (7.1) implies that

$$
\|U(t)\|_{H^{2}} \leq \quad \begin{gathered}
\|U(0)\|_{H^{2}}+ \\
C \int_{0}^{t}\left(1+\alpha_{\lambda}(s)+\frac{\|U(s)\|_{H^{2}}}{\sqrt{\lambda}}+\ln ^{+}\left(\|U(s)\|_{H^{2}}\right)\right)\|U(s)\|_{H^{2}} d s .
\end{gathered}
$$

Since the function $n \mapsto n+\alpha_{\lambda} n+n \ln ^{+}(n)+\frac{n^{2}}{\sqrt{\lambda}}$ is positive and increasing in $n$ the same inequality is true for $n_{2}(t)=\sup _{0 \leq s \leq t}\|U(t)\|_{H^{2}}$, that is

$$
n_{2}(t) \leq n_{2}(0)+C \int_{0}^{t}\left(1+\alpha_{\lambda}(s)+\frac{n_{2}(s)}{\sqrt{\lambda}}+\ln ^{+}\left(n_{2}(s)\right)\right) n_{2}(s) d s .
$$

This family of inequalities hold for all $t \in[0, T[$ and $\lambda \geq e$. The constant $C$ only depends on $\left\|M_{0}\right\|_{L^{2} \cap L^{\infty}}$ and the family of functions $\alpha_{\lambda}$ satisfy

$$
\left\|\alpha_{\lambda}\right\|_{L^{2}([0, T])} \leq C_{1} \sqrt{\ln \lambda}
$$

where $C_{1}$ only depends on $\|M(0)\|_{L^{\infty} \cap L^{2}},\|\operatorname{curl}(E, H)(0)\|_{L^{2}}$ and $T$.

To complete the proof of Proposition 7.1 consider $T_{1}<T$ and choose $\lambda$ such that $\sqrt{\lambda}=n_{2}\left(T_{1}\right)$ so that inequality (7.14) implies that, for $0 \leq t \leq T_{1}$,

$$
n_{2}(t) \leq \nu(t):=n_{2}(0)+C \int_{0}^{t}\left(\left(2+\alpha_{\lambda}(s)\right) n_{2}(s)+n_{2}(s) \ln ^{+} n_{2}(s)\right) d s .
$$


The function $\nu(t)$ is absolutely continuous on $\left[0, T_{1}\right]$ and its derivative is

$$
\nu^{\prime}(t)=C\left(2+\alpha_{\lambda}(t)+\ln ^{+} n_{2}(t)\right) n_{2}(t) \leq C\left(2+\alpha_{\lambda}(t)+\ln ^{+} \nu(t)\right) \nu(t) .
$$

In (7.13) and (7.15) one can increase $n_{2}(0)$ and therefore assume that $n_{2}(0)>1$, so that $\nu(t)>1$. Then the function $\ln \nu$ is absolutely continuous and its derivative is

$$
(\ln \nu)^{\prime}(t)=\frac{\nu^{\prime}(t)}{\nu(t)} \leq C\left(2+\alpha_{\lambda}(t)+\ln \nu(t)\right) .
$$

Therefore, for $t \leq T_{1}$, one gets

$$
\ln \nu(t) \leq e^{C t} \ln \nu(0)+\int_{0}^{t} e^{t-s}\left(2+\alpha_{\lambda}(s)\right) d s .
$$

Take $t=T_{1}$ in this estimate. Using (7.14) and recalling the choice $\lambda=n_{2}\left(T_{1}\right)^{2}$, (7.15) and (7.17) imply

$$
\ln n_{2}\left(T_{1}\right) \leq \ln \nu\left(T_{1}\right) \leq e^{C T} \ln n_{2}(0)+e^{C T} C_{1} \sqrt{2 T \ln n_{2}\left(T_{1}\right)} .
$$

Therefore

$$
\ln n_{2}\left(T_{1}\right) \leq 2 e^{C T} \ln n_{2}(0)+2 T e^{2 C T} C_{1}^{2}
$$

and Proposition 7.1 follows.

\section{Limit Strichartz-type estimates}

In this section we prove Proposition 6.3. Recall that the space dimension is equal to 3 . The proof follows the methods in $[\mathrm{GV}]$ or $[\mathrm{LS}]$, but we give the details to obtain the sharp bound $\sqrt{\ln (1+\lambda T)}$.

Consider $v \in C^{\infty}\left(\mathbf{R} ; \mathcal{S}\left(\mathbf{R}^{3}\right)\right)$ such that

$$
\square v=g, \quad v_{\mid t=0}=0, \quad \partial_{t} v_{\mid t=0}=0,
$$

Lemma 8.1 Suppose that the support of the spatial Fourier transform $\hat{g}(t, \xi)$ of $g$ is contained in the ball $\{|\xi| \leq \lambda\}$. Then for all $t \geq 0$,

$$
\left\{\begin{array}{c}
\|v(t)\|_{L^{2}}^{2} \leq \frac{5}{2 \pi^{2}} \ln (1+\lambda t) \int_{0}^{t}\|g(s)\|_{L^{1}}^{2} d t \\
\left\|\partial_{t} v(t)\right\|_{\dot{H}^{-1}}^{2} \leq \frac{5}{2 \pi^{2}} \ln (1+\lambda t) \int_{0}^{t}\|g(s)\|_{L^{1}}^{2} d t .
\end{array}\right.
$$

Proof. We consider first the case $\lambda=1$. The general case follows using dilations.

1) Suppose that

$$
\text { support } \hat{g}(t, \xi) \subset\{|\xi| \leq 1\} .
$$

The solution to (8.1) satisfies

$$
\hat{v}(t, \xi)=\int_{0}^{t} \sin ((t-s)|\xi|) \hat{g}(s, \xi) \frac{d s}{|\xi|},
$$


and

$$
\frac{\partial_{t} \hat{v}(t, \xi)}{|\xi|}=\int_{0}^{t} \cos ((t-s)|\xi|) \hat{g}(s, \xi) \frac{d s}{|\xi|} .
$$

Thus

$$
\|v(t)\|_{L^{2}}^{2}=\int_{0}^{t} \int_{0}^{t} \int_{\mathbf{R}^{3} \times \mathbf{R}^{3}} K_{-}(\tau, \sigma, x-y) g(\sigma, y) \overline{g(\tau, x)} d x d y d \tau d \sigma
$$

where, taking the support condition (8.3) into account,

$$
(2 \pi)^{3} K_{-}(\tau, \sigma, z):=\int_{|\xi| \leq 1} \sin ((t-\sigma)|\xi|) \sin ((t-\tau)|\xi|) e^{-i z \cdot \xi} \frac{d \xi}{|\xi|^{2}} .
$$

Similarly,

$$
\left\|\frac{\partial_{t} v(t)}{|\xi|}\right\|_{L^{2}}^{2}=\int_{0}^{t} \int_{0}^{t} \int_{\mathbf{R}^{3} \times \mathbf{R}^{3}} K_{+}(\tau, \sigma, x-y) g(\sigma, y) \overline{g(\tau, x)} d x d y d \tau d \sigma
$$

with

$$
(2 \pi)^{3} K_{+}(\tau, \sigma, z):=\int_{|\xi| \leq 1} \cos ((t-\sigma)|\xi|) \cos ((t-\tau)|\xi|) e^{-i z \cdot \xi} \frac{d \xi}{|\xi|^{2}} .
$$

Introduce

$$
M(\lambda, z)=\int_{|\xi| \leq 1} \cos (\lambda|\xi|) e^{-i z \cdot \xi} \frac{d \xi}{|\xi|^{2}}
$$

It follows that

$$
(2 \pi)^{3} K_{ \pm}(\tau, \sigma, z)=\frac{1}{2}(M(\tau-\sigma, z) \pm M(2 t-\sigma-\tau, z)) .
$$

In order to apply Schur's Lemma to (8.4) and (8.6), we need sharp bounds for $\sup _{z}\left|K_{ \pm}(\tau, \sigma, z)\right|$, hence, in view of (8.9), of $\sup _{z}|M(\lambda, z)|$. From (8.8) one gets first that, for all $\lambda, z$,

$$
|M(\lambda, z)| \leq 4 \pi
$$

Note that $M$ is real and rotation invariant in $z$. Taking polar coordinates for $\xi$, one obtains that

$$
M(\lambda, z)=2 \pi \int_{0}^{1} \int_{-1}^{1} \cos (\lambda r) e^{i|z| r \omega} d r d \omega=\pi \int_{-1}^{1} \frac{\sin (|z| \omega+\lambda)}{|z| \omega+\lambda}+\frac{\sin (|z| \omega-\lambda)}{|z| \omega-\lambda} d \omega .
$$

Writing

$$
\int_{-1}^{1} \frac{\sin (|z| \omega \pm \lambda)}{|z| \omega \pm \lambda} d \omega=\frac{1}{|z|} \int_{-|z| \pm \lambda}^{|z| \pm \lambda} \frac{\sin (a)}{a} d a
$$

it follows that

$$
|M(\lambda, z)| \leq \frac{4 \pi S i(\pi)}{|z|}<\frac{8 \pi}{|z|}
$$


since the function $\operatorname{Si}(x)=\int_{0}^{x} \frac{\sin (a)}{a} d a$ satisfies $|S i(x)| \leq S i(\pi)<2, x \in \mathbf{R}$. From (8.11) one also gets

$$
\sup _{|z| \leq \frac{|\lambda|}{2}}|M(\lambda, z)| \leq \frac{8 \pi}{|\lambda|}
$$

which, with (8.12) and (8.10), yields

$$
\sup _{z}|M(\lambda, z)| \leq \frac{20 \pi}{1+|\lambda|}
$$

To estimate (8.4) and (8.6) we note that

$$
\begin{aligned}
& \left|\int_{0}^{t} \int_{0}^{t} \int_{\mathbf{R}^{3} \times \mathbf{R}^{3}} K_{ \pm}(\tau, \quad \sigma, x-y) g(\sigma, y) \overline{g(\tau, x)} d x d y d \tau d \sigma\right| \leq \\
& \left(\sup _{\tau} \int_{0}^{t} \sup _{z}\left|K_{ \pm}(\tau, \sigma, z)\right| d \sigma\right) \int_{0}^{t}\|g(s)\|_{L^{1}}^{2} d s .
\end{aligned}
$$

Using (8.9) and (8.14), one has

$$
(2 \pi)^{3} \sup _{z}\left|K_{ \pm}(\tau, \sigma, z)\right| \leq \frac{10 \pi}{1+|\tau-\sigma|}+\frac{10 \pi}{1+|2 t-\tau-\sigma|} .
$$

Hence

$$
(2 \pi)^{3} \int_{0}^{t} \sup _{z}\left|K_{ \pm}(\tau, \sigma, z)\right| \leq 10 \pi(\ln (1+\tau)+\ln (1+2 t-\tau))
$$

and

$$
\sup _{\tau} \int_{0}^{t} \sup _{z}\left|K_{ \pm}(\tau, \sigma, z)\right| d \sigma \leq \frac{5}{2 \pi^{2}} \log (1+t)
$$

Substituting this estimate in (8.15), equalities (8.4) and (8.6) yield

$$
\left\|\partial_{t} v(t)\right\|_{\dot{H}^{-1}}^{2} \leq \frac{5}{2 \pi^{2}} \ln (1+t) \int_{0}^{t}\|g(s)\|_{L^{1}}^{2} d s
$$

and

$$
\|v(t)\|_{L^{2}}^{2} \leq \frac{5}{2 \pi^{2}} \ln (1+t) \int_{0}^{t}\|g(s)\|_{L^{1}}^{2} d s
$$

2) Suppose next that $\hat{g}(t, \xi)$ is supported in $\{|\xi| \leq \lambda\}$. Introduce

$$
g_{\lambda}(t, x):=\frac{1}{\lambda^{2}} g\left(\frac{t}{\lambda}, \frac{x}{\lambda}\right), \quad v_{\lambda}(t, x):=v\left(\frac{t}{\lambda}, \frac{x}{\lambda}\right) .
$$

Then

$$
\square v_{\lambda}=g_{\lambda}, \quad v_{\lambda \mid t=0}=0, \quad \partial_{t} v_{\lambda \mid t=0}=0,
$$

and $\hat{g}_{\lambda}(t, \xi)=\lambda \hat{g}(t / \lambda, \lambda \xi)$ is supported in $\{|\xi| \leq 1\}$. Thus (8.16) (8.17) apply to $v_{\lambda}$ and $g_{\lambda}$ and (8.2) follows. 
Proof of Proposition 6.3. Consider $f \in C^{\infty}\left(\mathbf{R} ; \mathcal{S}\left(\mathbf{R}^{3}\right)\right), u_{0} \in \mathcal{S}\left(\mathbf{R}^{3}\right)$ and $u_{1} \in$ $\mathcal{S}\left(\mathbf{R}^{3}\right)$. Let $u \in L_{l o c}^{\infty}\left(\mathbf{R}^{+} ; L^{2}\left(\mathbf{R}^{3}\right)\right.$ denote the solution to

$$
\square u=f, \quad u_{\mid t=0}=u_{0}, \quad \partial_{t} u_{\mid t=0}=u_{1} .
$$

Recall from (2.9) that $S^{\lambda}=\varphi\left(\lambda^{-1} D_{x}\right)$ where $\left.\varphi \in C_{0}^{\infty}\left(\mathbf{R}^{3}\right)\right)$ is real, equal to 1 on $|\xi| \leq 1$ and is supported in $|\xi| \leq 2$. We show now there is a constant $C$, depending on $\varphi$ but not on $T$ and $u$, such that

$$
\sqrt{\int_{0}^{T}\left\|S^{\lambda}(u)(t)\right\|_{\infty}^{2} d t} \leq C \sqrt{\log (1+2 \lambda T)}\left(\left\|u_{0}\right\|_{\dot{H}^{1}}+\left\|u_{1}\right\|_{2}+\int_{0}^{T}\|f(t)\|_{L^{2}} d t\right) .
$$

Note that the left hand side of (8.20) is well defined. One has

$$
\left\|S^{\lambda}(u)(t)\right\|_{L^{2}\left([0, T] ; L^{\infty}\right)}=\sup _{\|g\|_{L^{2}\left([0, T] ; L^{1}\right) \leq 1}} \int_{\Omega_{T}} S^{\lambda}(u) g d x d t,
$$

where $\Omega_{T}=[0, T] \times \mathbf{R}^{3}$ and the functions $g$ are supposed smooth. To any such $g$ corresponds a unique $v$ solution on $\{t \leq T\} \times \mathbf{R}^{3}$ to

$$
\square v=g, \quad v_{\mid t=T}=0, \quad \partial_{t} v_{\mid t=T}=0 .
$$

Since $\varphi$ is real, we get

$$
\int_{\Omega_{T}} S^{\lambda}(u) g d x d t=\int_{\Omega_{T}} u S^{\lambda}(g) d x d t .
$$

Commuting $S^{\lambda}$ and $\square$ yields

$$
\square S^{\lambda} v=S^{\lambda} g, \quad S^{\lambda} v_{\mid t=T}=0, \quad \partial_{t} S^{\lambda} v_{\mid t=T}=0 .
$$

Thus, integrating by part, one obtains

$$
\begin{gathered}
\left|\int_{\Omega_{T}} S^{\lambda}(u) g d x d t\right| \leq\|f\|_{L^{1}\left([0, T] ; L^{2}\right)}\left\|S^{\lambda}(v)\right\|_{L^{\infty}\left([0, T] ; L^{2}\right)}+ \\
\left\|u_{0}\right\|_{\dot{H}^{1}}\left\|\partial_{t} S^{\lambda}(v)(0)\right\|_{\dot{H}^{-1}}+\left\|u_{1}\right\|_{L^{2}}\left\|\partial_{t} S^{\lambda}(v)(0)\right\|_{L^{2}} .
\end{gathered}
$$

Using (8.2) for $S^{\lambda}(v)$ whose spectrum belongs to $|\xi| \leq 2 \lambda$, it follows that

$$
\begin{gathered}
\left|\int_{\Omega_{T}} S^{\lambda}(u) g d x d t\right| \leq \\
\left\|S^{\lambda}(g)\right\|_{L^{2}\left([0, T] ; L^{1}\right)} \sqrt{\frac{5}{2 \pi^{2}} \log (1+2 \lambda T)}\left(\left\|u_{0}\right\|_{\dot{H}^{1}}+\left\|u_{1}\right\|_{L^{2}}+\|f\|_{L^{1}\left([0, T] ; L^{2}\right)}\right) .
\end{gathered}
$$

To conclude note that the operators $S^{\lambda}$ are uniformly bounded from $L^{1}\left(\mathbf{R}^{3}\right)$ to $L^{1}\left(\mathbf{R}^{3}\right)$. Thus $\left\|S^{\lambda}(g)\right\|_{L^{2}\left([0, T] ; L^{1}\right)} \leq C\|(g)\|_{L^{2}\left([0, T] ; L^{1}\right)} \leq C$ for some $C$ that only depends on $\varphi$. With (8.21), (8.24) implies (8.20) and Proposition 6.3. 


\section{References}

[1] Bony J-M., Calcul symbolique et propagation des singularités pour les équations aux dérivées partielles non linéaires, Ann. Scient. de l'E.N.S.14, 1981, pp 209-246.

[2] Carbou G., Fabrie P., Comportement asymptotique des solutions faibles des équations de Landau-Lifshitz, C. R. Acad. Sci. Paris, t. 325, Série I, p. 717$720,1997$.

[3] Coiffman, Meyer Y, Au delà des opérateurs pseudodifférentiels, Astérisque $57,1978$.

[4] Donnat P., Rauch J., Global solvability of the Maxwell-Bloch equations from nonlinear optics, Arch. Rat. Mech. Anal., to appear

[5] Gérard P., Microlocal defect measures, Comm. Partial Diff. Equ., 16, 1991, pp 1761-1794.

[6] Ginibre J., Velo G., Generalized Strichartz inequalities for the wave equation, J. Funct. Anal., 133 (1995), n 1, 50-68.

[7] Joly J.-L., Métivier G., Rauch J., Global solvability of the anharmonic oscillator model from nonlinear optics, SIAM J.Math.Anal., 27, 1996, pp 905-913.

[8] Joly J.-L., Métivier G., Rauch J., Solutions globales du système de Maxwell dans un milieu ferro magnétique, Séminaire École Polytechnique, 1997.

[9] Joly J.-L., Métivier G., Rauch J., Propagation des ondes électromagnétiques en présence d'un matériau ferromagnétique, pp. 85-99. Actes du 29ème Congrès d'Analyse Numérique : CANum'97, ESAIM: Proceedings, Vol. x, 1998, http://www.emath.fr/proc/Vol.X/

[10] Joly P., Vacus O., Mathematical and numerical studies of 1D nonlinear ferromagnetic materials, Rapport INRIA, n 3024, 1996.

[11] Joly P., Vacus O., Maxwell's equations in a 1D ferromagnetic medium: Existence and uniqueness of strong solutions, Rapport INRIA, n 3052, 1996.

[12] Klainerman S., Machedon M., Space-time estimates for null-forms and the local existence theorem, Com. Pure Appl. Math., 46 (1993), 1221-1268.

[13] Landau L., Lifshitz E., Physik A, Soviet Union 8, 1935, 153.

[14] Landau L., Lifshitz E., Électrodunamique des milieux continus, cours de physique théorique, t. 8, éditions Mir, Moscou, 1969.

[15] Lindblad H., Counterexamples to local existence for semi-linear wave equation, Amer. J. Math. 118 (1996), n 1, 1-16. 
[16] Lindblad H, Sogge C.D., On existence and scattering with minimal regularity for semilinear wave equations, J. Funct. Anal., 130, 1995, pp 357-426.

[17] Stein, E. Singular integrals and differentiability properties of functions, Princeton University Press, 1970.

[18] Tartar L., H-measures, a new approach for studying homogeneization, oscillations and concentrations effects in partial differential equations, Poc. Roy. Soc. Edinburgh, 115 (A), 1990, pp193-230.

[19] Triebel H., Theory of functions spaces, Birkhäuser, Basel, 1983.

[20] Visintin, A. On Landau-Lifshitz equations for ferromagnetism, Japan J. of Appl. Math., 2, No. 1, 1985, 69-84.

J.L. Joly

MAB, Université Bordeaux I

F-33405 Talence, France

e-mail : joly@math.u-bordeaux.fr

G. Métivier

IRMAR, Université Rennes I

F-35042 Rennes, France

e-mail : metivier@univ-rennes1.fr

J. Rauch

Department of Mathematics

University of Michigan

Ann Arbor MI 48109, USA

Communicated by J. Bellissard

submitted 21/01/98; accepted 10/04/98 